\title{
Dealing with uncertainty in model updating for damage assessment: a review
}

\author{
Ellen Simoen ${ }^{\mathrm{a}}$, Guido De Roeck ${ }^{\mathrm{a}}$, Geert Lombaert ${ }^{\mathrm{a}, *}$ \\ ${ }^{a} K U$ Leuven, Department of Civil Engineering, Kasteelpark Arenberg 40 box 2448, B-3001 Leuven, Belgium
}

\begin{abstract}
In structural engineering, model updating is often used for non-destructive damage assessment: by calibrating stiffness parameters of finite element models based on experimentally obtained (modal) data, structural damage can be identified, quantified and located. However, the model updating problem is an inverse problem prone to ill-posedness and ill-conditioning. This means the problem is extremely sensitive to small errors, which may potentially detract from the method's robustness and reliability. As many errors or uncertainties are present in model updating, both regarding the measurements as well as the employed numerical model, it is important to take these uncertainties suitably into account. This paper aims to provide an overview of the available approaches to this end, where two methods are treated in detail: a non-probabilistic fuzzy approach and a probabilistic Bayesian approach. These methods are both elaborated for the specific case of vibration-based finite element model updating for damage assessment purposes.
\end{abstract}

Keywords: damage assessment, uncertainty quantification, structural dynamics, model updating, Bayesian inference, fuzzy set theory

\section{Contents}

1 Introduction 2

1.1 Why model updating? . . . . . . . . . . . . . . . . . . . . . . . . . . 2

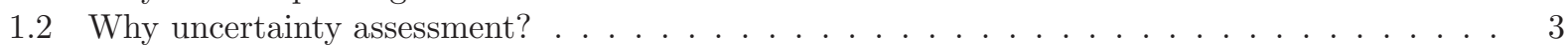

1.3 An illustrative example of FE model updating . . . . . . . . . . . . . . . . . . . . . . . . 3

2 Deterministic FE model updating 4

2.1 Models, model classes and model updating . . . . . . . . . . . . . . . . . . . . . . . . . . 4

2.2 Vibration-based FE model updating . . . . . . . . . . . . . . . . . . . . . . . . . . . . . . 5

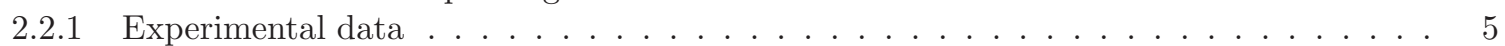

2.2 .2 Model class and computed data . . . . . . . . . . . . . . . . . . . . . . 6

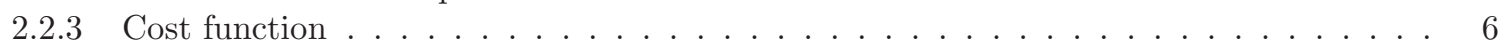

2.3 Solution and ill-posedness of the optimization problem . . . . . . . . . . . . . . . . . . . 7

2.4 RC beam example . . . . . . . . . . . . . . . . . . . . . . . . . . . . . . . 8

\begin{tabular}{|lll}
\hline 3 & Uncertainty in model updating & 11
\end{tabular}

3.1 Uncertainty related to the prediction model . . . . . . . . . . . . . . . . . . . . . . 11

3.2 Uncertainty related to the experimental data . . . . . . . . . . . . . . . . . . . . . 12

3.3 Combining measurement and model uncertainty . . . . . . . . . . . . . . . . . . . . . . . . 12

3.4 Modeling of uncertainties . . . . . . . . . . . . . . . . . . . . . . . . . 13

\footnotetext{
* Corresponding author

Email address: geert.lombaert@bwk.kuleuven.be (Geert Lombaert) Postprint submitted to Mechanical Systems and Signal Processing

Published version: E. Simoen, G. De Roeck, and G. Lombaert. Dealing with uncertainty in model updating for damage assessment: a review. Mechanical Systems and Signal Processing, 56-57:123-149, 2015. http://dx.doi.org/10.1016/j.ymssp.2014.11.001
} 


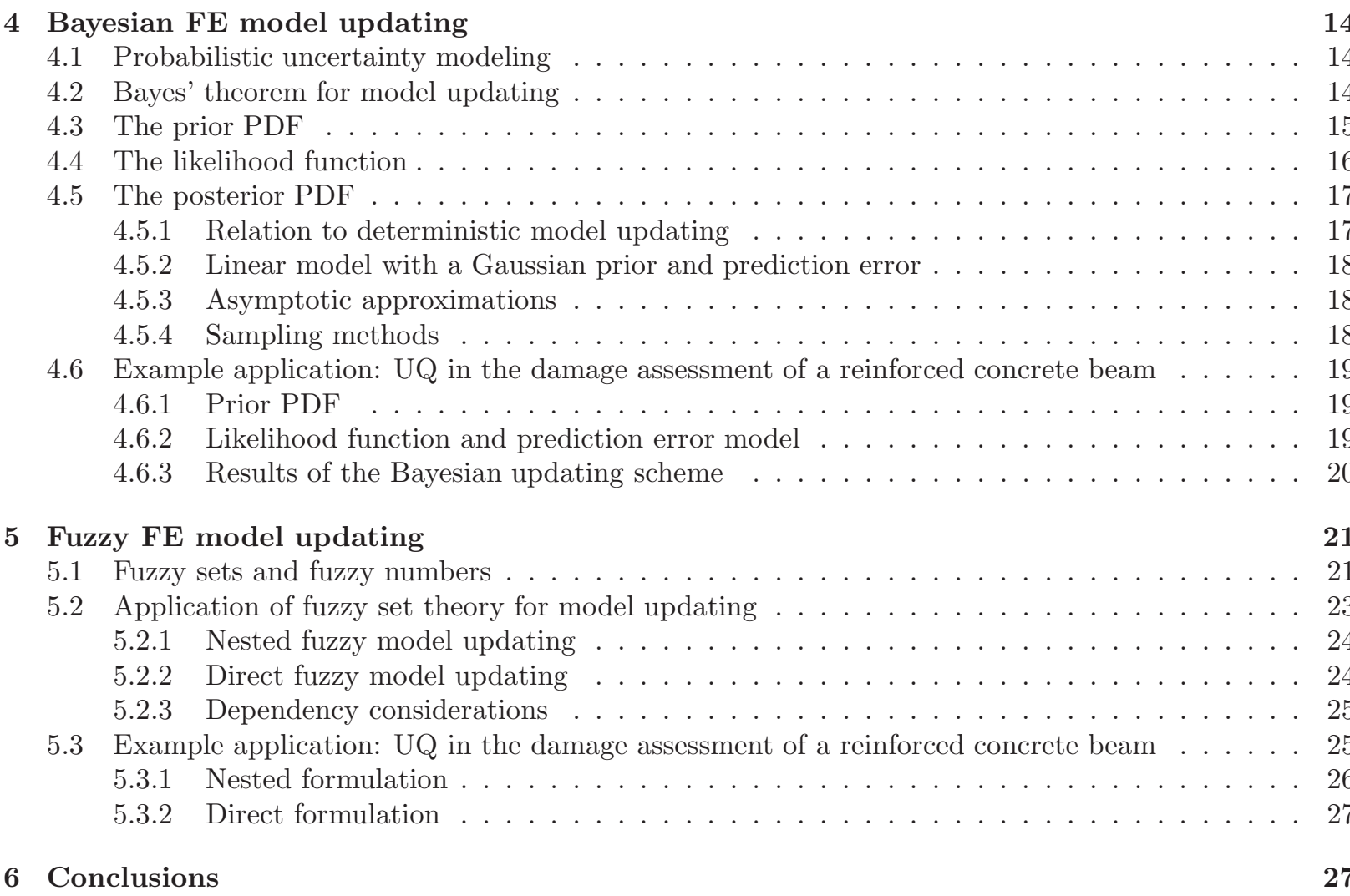

\section{Introduction}

\subsection{Why model updating?}

In practically all areas of science and engineering, numerical or mathematical models are used to simulate the behavior of real systems. The purposes of these numerical models vary widely, but can generally be classified into three main categories: analysis, prediction, and design. In civil and structural engineering, finite element (FE) models are most often used to analyze e.g. the internal forces and displacements of structures in several limit states, or to predict vibration responses due to dynamic loading such as earthquakes, wind and traffic. These FE analyses can also be used to design structural components or complete structures. It goes without saying that for all the above purposes, the validity of the adopted numerical models is imperative. There are, however, always numerous unknown or uncertain system properties (e.g. regarding material properties, geometric properties, boundary conditions, load conditions) for which inevitably conjectures have to be made. Moreover, due to a lack of knowledge or other restrictions, often simplifying modeling assumptions regarding the model structure are required or implicitly made. These issues may detract from the quality and accuracy of the numerical model and its purposes.

This has led to the development of model updating techniques, also referred to as model calibration or, in more generic terms, parameter identification or estimation. Generally speaking, model updating aims to reconstruct or calibrate unknown system properties which appear as parameters in numerical models, based on actually observed behavior of the system of interest. In a structural mechanics context, often (processed) data acquired in vibration experiments (i.e. acceleration time histories, frequency response functions, natural frequencies and mode shape displacements, modal strains or curvatures, modal flexibilities, etc.) are deemed most suited for FE model updating purposes, as they provide detailed information regarding the global and local behavior of the structure of interest, and can be measured in an operational state of the structure. Standard reference works on deterministic vibration-based FE model updating include those of Mottershead 
and Friswell [1, 2], Fritzen et al. [3] and Imregun and Visser [4]; for a comprehensive overview of FE model updating in civil engineering applications, the reader is referred to the work of Teughels [5].

Structural FE model updating serves a wide array of purposes; it can for instance be applied for design verification and validation, to obtain improved predictions of structural response quantities, or simply to identify unknown system characteristics. One of the most prominent application areas of vibration-based FE model updating is found in structural health monitoring (SHM). The basic principle behind this consists in assuming that localized structural damage results in a local reduction of stiffness. As such, updating stiffness parameters of the FE model in several damage states provides a (non-destructive) means to thoroughly and accurately investigate the condition of the structure. Up-to-date reference works on SHM can be retrieved in 6 6] ; for vibration-based SHM, the reader is referred to the extensive literature reviews by Doebling et al. 10, 11], and Carden and Fanning [12], and recent works by Brownjohn et al. [13] and Deraemaeker and Worden 14]. Rytter 15] describes a four-tiered approach to damage assessment: (1) damage detection or identification, (2) damage localization, (3) damage quantification and (4) prognosis of the remaining service life of the structure. Application of FE model updating directly allows damage assessment up to level 3; it provides a means to detect the presence of damage, locate the damage along the structure and quantify the extent of the damage. Notwithstanding the importance of the first three levels, the fourth level is the ultimate goal of any SHM procedure, but it is also by far the most challenging.

\subsection{Why uncertainty assessment?}

Model updating problems are inverse problems, as they aim to invert the standard "forward" relationship between parameters and output of a model; the objective is instead to obtain the parameters that produce a certain given output. More specifically, deterministic model updating aims to find the optimal parameters of a numerical model such that the best possible fit is obtained between the model output and the observed data. This is usually accomplished by formulating the problem as a constrained optimization problem, where the objective is to minimize the discrepancy between computed and measured data.

In many cases, however, this optimization problem is prone to ill-posedness and ill-conditioning, meaning that the existence, uniqueness and stability (with respect to small errors) of a solution of the inverse problem cannot be guaranteed. This is a non-negligible issue in model updating, as the problem is always subject to measurement and modeling errors, which give rise to uncertain data and model predictions. Accounting for these uncertainties is therefore an important and indispensable undertaking in model updating. Applying uncertainty quantification (UQ) in model updating not only allows assessing the effect of uncertainty on the updated model parameters, but moreover provides a means to evaluate the uncertainties on derived quantities such as response predictions. Ultimately, this allows assessing the robustness of decisions made based on these predictions and, as such, the degree of usability the model [16].

Over the last decades, UQ in science and engineering has gained substantially in interest, leading to the development of a huge amount of UQ approaches in various scientific domains. The question that arises is whether (and how) these approaches can be applied for efficient UQ in vibration-based FE model updating, and which are most suited to which end purpose. This will constitute the main topic of the current review paper, where the focus will be placed on civil engineering SHM applications. In the following section, the specifics of deterministic FE model updating are recalled and the importance of accounting for uncertainty is pointed out; the types of uncertainty occurring in model updating are discussed in section 3 . Out of the wide array of available UQ approaches, two are treated in detail in this paper: a probabilistic Bayesian approach (section (4) and a non-probabilistic fuzzy approach (section [5). Arguably, these two methods constitute the most popular of their corresponding domains.

\subsection{An illustrative example of FE model updating}

Throughout this paper, a simple but instructive engineering application will serve as a running example to illustrate the workings of both UQ methods. The application concerns the damage assessment of a reinforced concrete $(\mathrm{RC})$ beam with a length of $6 \mathrm{~m}$ and dimensions as shown in figure 1a, The beam was built in a laboratory environment, and controlled damage was induced by applying a static load of $25 \mathrm{kN}$ at two thirds of the length of the beam (figure 1b). After applying the damage, free-free vibration tests 


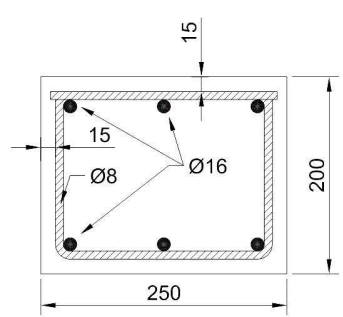

(a)

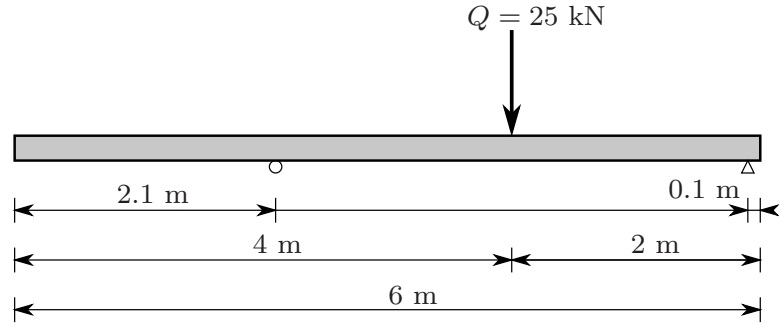

(b)

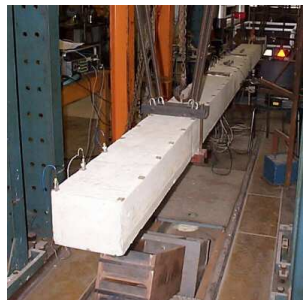

(c)

Figure 1: (a) Cross-section of the reinforced concrete beam, (b) set-up of static loading and (c) image of vibration testing [21].

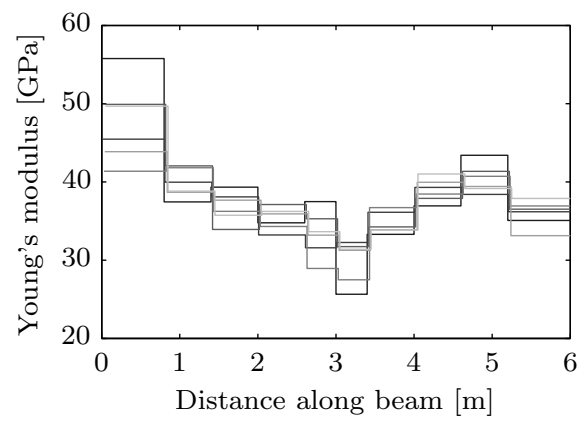

(a)

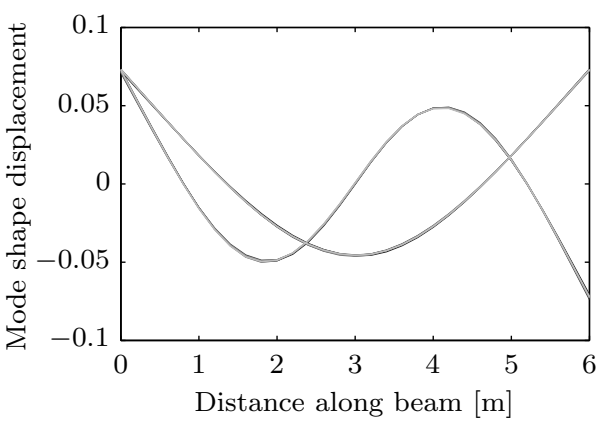

(b)

Figure 2: (a) Six different stiffness patterns along a reinforced concrete beam that yield almost identical modal properties, as demonstrated in (b) where the corresponding first two predicted bending modes of the beam are shown.

(figure 1c) were performed which allowed identifying a set of modal characteristics (i.e. natural frequencies and mode shapes) of the beam in its damaged condition. The objective of this example is to use these measurements to assess the structural damage occurring in the RC beam through FE model updating, by calibrating the stiffness pattern along the beam.

Figure 2 illustrates the ill-conditioning issues for this RC beam example. Figure 2a shows six different stiffness patterns along the beam, and figure $2 \mathrm{~b}$ shows the first two computed bending modes corresponding to the patterns in figure 2a, It is clear that all the stiffness patterns lead to virtually identical low order bending modes, making it very difficult to uniquely and accurately determine the stiffness values when only these mode shapes are given. Moreover, it is clear that small variations in the measurements or in the model predictions could lead to widely varying updating results.

\section{Deterministic FE model updating}

Before discussing uncertainties and means to deal with uncertainty in model updating, a brief overview is given of vibration-based deterministic FE model updating, starting with a short formal description of model classes and model updating.

\subsection{Models, model classes and model updating}

Most generally, a model is defined as a description of the assumed relationship between input and output variables of a system, taking the (known or assumed) properties of that system into account. The inputoutput behavior of the system can then be described by its model or transfer operator $\mathbf{G}_{\mathrm{M}}$. This model operator is specified in terms of a finite number of numerical model parameters $\boldsymbol{\theta}_{\mathrm{M}}$, so that the output response $\mathbf{y}$ due to any input vector $\mathbf{x}$ can be written in a general way as $\mathbf{y}=\mathbf{G}_{\mathbf{M}}\left(\mathbf{x}, \boldsymbol{\theta}_{\mathrm{M}}\right)$. The outputs of interest can relate to directly measurable system response quantities (accelerations, displacements, strains, 
etc.) or derived features such as frequency response functions. It is often convenient to work with outputs that are independent from the input $\mathbf{x}$ (such as modal properties, see section 2.2.1), as the input is in many practical applications not precisely known. In the remainder of this text, this will be presumed the case and the input $\mathbf{x}$ is dropped from the notations: $\mathbf{y}=\mathbf{G}_{\mathrm{M}}\left(\boldsymbol{\theta}_{\mathrm{M}}\right)$. It is essential to note that, as the parameter vector $\boldsymbol{\theta}_{\mathrm{M}}$ ranges over a subset $D_{\mathrm{M}}$ of $\mathbb{R}^{N_{\mathrm{M}}}, \mathbf{G}_{\mathrm{M}}\left(\boldsymbol{\theta}_{\mathrm{M}}\right)$ represents not a single model but a set or class of models $\mathcal{M}_{\mathrm{M}}$. Each model in the structural model class $\mathcal{M}_{\mathrm{M}}=\left\{\mathcal{M}_{\mathrm{M}}\left(\boldsymbol{\theta}_{\mathrm{M}}\right) \mid \boldsymbol{\theta}_{\mathrm{M}} \in D_{\mathrm{M}}\right\}$ provides a mapping from the model parameter space $D_{\mathrm{M}}$ to the output space $\mathbb{R}^{N}$ as follows:

$$
\mathcal{M}_{\mathrm{M}} \quad: \quad D_{\mathrm{M}} \subseteq \mathbb{R}^{N_{\mathrm{M}}} \rightarrow \mathbb{R}^{N} \quad: \quad \boldsymbol{\theta}_{\mathrm{M}} \mapsto \mathbf{y}=\mathbf{G}_{\mathrm{M}}\left(\boldsymbol{\theta}_{\mathrm{M}}\right)
$$

Deterministic model updating now consists in determining an optimal set of model parameters $\boldsymbol{\theta}_{\mathrm{M}}^{*}$ that minimizes the misfit between some experimental data, denoted as $\overline{\mathbf{d}}$, and model predictions $\mathbf{G}_{\mathrm{M}}\left(\boldsymbol{\theta}_{\mathrm{M}}\right)$. This discrepancy is represented by the so-called misfit or cost function $F$, so that the model updating problem is equivalent to the following optimization problem:

$$
\boldsymbol{\theta}_{\mathrm{M}}^{*}=\underset{\boldsymbol{\theta}_{\mathrm{M}} \in D_{\mathrm{M}}}{\arg \min } F\left(\mathbf{G}_{\mathrm{M}}\left(\boldsymbol{\theta}_{\mathrm{M}}\right), \overline{\mathbf{d}}\right)=\underset{\boldsymbol{\theta}_{\mathrm{M}} \in D_{\mathrm{M}}}{\arg \min } F\left(\boldsymbol{\theta}_{\mathrm{M}}\right)
$$

where the latter equality takes into account that for a given model class and a given set of experimental data $\overline{\mathbf{d}}$, the cost function $F$ only depends on the model parameters $\boldsymbol{\theta}_{\mathrm{M}}$. In many cases, the optimization problem

(2) is formulated as a constrained optimization problem, where the constraints depend on the nature of the considered model updating problem and on the information that is available on the updating parameters. For instance, inequality constraints can be set when it is known that the parameters lie within a certain interval.

\subsection{Vibration-based FE model updating}

\subsubsection{Experimental data}

The experimental data $\overline{\mathbf{d}}$ used for damage assessment through FE model updating most often consist of measurements obtained during dynamic vibration experiments, where a structure is equipped with a set of measurement sensors (e.g. accelerometers, strain gauges, fibre-optic sensors, etc.) which register the system's response due to some dynamic excitation. However, response time domain data such as accelerations are not often used directly for model updating, as this would require the precise knowledge of the input excitation - a condition seldom fulfilled in civil engineering applications. Therefore, most often use is made of modal characteristics, which are extracted from the measured response time histories using modal analysis techniques [22]. Modal data are in general rich in information content, and can be obtained in an operational state of the structure, meaning that no external excitation (e.g. drop weights, shaker) needs to be applied or known. A drawback of operational testing versus forced excitation testing is that modal masses cannot be uniquely determined.

Several types of modal data can be used. Most basically, model updating can be performed based only on changes of $N_{m}$ natural frequencies $\bar{f}_{r}$ or eigenvalues $\bar{\lambda}_{r}=\left(2 \pi \bar{f}_{r}\right)^{2}$, which are well known to be significantly affected by changes in structural stiffness, and can be measured fairly accurately. However, eigenfrequencies provide only global information on the structure's condition. In order to allow for effective damage localization, mode shape displacements $\bar{\phi}_{r} \in \mathbb{R}^{N_{o}}$ can be added to the data set; this constitutes one of the most common data configurations for FE model updating. However, mode shape displacements are usually more difficult to measure accurately, and are not extremely sensitive to moderate changes in structure stiffness (as demonstrated in figure 2). Moreover, obtaining a sufficiently informative mode shape data set often necessitates a large number of sensors. Therefore, the (additional) use of other derived modal characteristics is advocated by many authors. The most popular quantities include modal flexibilities 2326], modal curvatures [27, 28], and modal strain energies [29, 30], which are considered to be much more sensitive to changes in stiffness. All modal features discussed here can be used for model updating, both separately or combined.

Instead of modal domain data, frequency domain data such as frequency response functions (FRFs) can be employed directly for structural model updating as well [31, 32]. Even though this technique requires the 
knowledge of the excitation forces (and thus require forced excitations), FRFs are sometimes preferred over modal data as additional errors due to the modal analysis procedure are avoided [33]. The use of FRFs in the modal updating process requires, in addition, an estimation of the modal damping ratios, as these can in general not be very accurately predicted.

\subsubsection{Model class and computed data}

The dynamic behavior of a structural FE model class with $N_{d}$ degrees of freedom is described by the following set of $N_{d}$ differential equations, relating the displacement vector $\mathbf{u}(t) \in \mathbb{R}^{N_{d}}$ and the excitation vector $\mathbf{f}(t) \in \mathbb{R}^{N_{d}}$ :

$$
\mathbf{M}\left(\boldsymbol{\theta}_{\mathrm{M}}\right) \ddot{\mathbf{u}}(t)+\mathbf{C}\left(\boldsymbol{\theta}_{\mathrm{M}}\right) \dot{\mathbf{u}}(t)+\mathbf{K}\left(\boldsymbol{\theta}_{\mathrm{M}}\right) \mathbf{u}(t)=\mathbf{f}(t)
$$

where $\mathbf{M}\left(\boldsymbol{\theta}_{\mathrm{M}}\right), \mathbf{C}\left(\boldsymbol{\theta}_{\mathrm{M}}\right)$ and $\mathbf{K}\left(\boldsymbol{\theta}_{\mathrm{M}}\right) \in \mathbb{R}^{N_{d} \times N_{d}}$ represent the global mass, damping and stiffness matrices, respectively. These matrices are constructed based on the characteristics of the elements describing the structure. Using the equations of motion (3), the structural model class $\mathcal{M}_{\mathrm{M}}$ can be constructed, depending on the output quantities of interest. Time and frequency responses to an excitation $\mathbf{f}(t)$ can be computed directly from equation (3) and its frequency domain transform; modal data follow from the solution of the (undamped) eigenvalue equation $\mathbf{K}\left(\boldsymbol{\theta}_{\mathrm{M}}\right) \boldsymbol{\Phi}=\mathbf{M}\left(\boldsymbol{\theta}_{\mathrm{M}}\right) \boldsymbol{\Phi} \boldsymbol{\Lambda}$. In this equation, matrix $\boldsymbol{\Phi} \in \mathbb{R}^{N_{d} \times N_{d}}$ collects the $N_{d}$ eigenvectors $\phi_{r} \in \mathbb{R}^{N_{d}}$ that correspond to the eigenvalues $\lambda_{r}=\left(2 \pi f_{r}\right)^{2}$ located on the diagonal of $\boldsymbol{\Lambda}$.

The model class is represented using its model operator $\mathbf{G}_{\mathrm{M}}$, so that the set of computed output data is denoted as $\mathbf{y}=\mathbf{G}_{\mathrm{M}}\left(\boldsymbol{\theta}_{\mathrm{M}}\right)$.

FE model parameterization. The choice of a suitable set of updating parameters $\boldsymbol{\theta}_{\mathrm{M}}$ is a non-trivial task: the set of selected parameters should be able to represent the unknown structural properties, but the number of updating parameters should also remain limited in order to avoid ill-conditioning problems (section 2.3). When using model updating for damage assessment purposes, the basic premise is that structural damage results in a local loss of stiffness; therefore the model parameterization can usually be limited to a parameterization of the stiffness matrix and, occasionally, the mass matrix. Although nonlinear parameterizations are possible, a linear relation between the system matrices and the model parameters is usually adopted as follows:

$$
\mathbf{K}\left(\boldsymbol{\theta}_{\mathrm{M}}\right)=\mathbf{K}_{0}+\sum_{j=1}^{N_{\mathrm{M}}} \theta_{\mathrm{M}, j} \mathbf{K}_{j} \quad \text { and } / \text { or } \quad \mathbf{M}\left(\boldsymbol{\theta}_{\mathrm{M}}\right)=\mathbf{M}_{0}+\sum_{j=1}^{N_{\mathrm{M}}} \theta_{\mathrm{M}, j} \mathbf{M}_{j}
$$

where each $\mathbf{K}_{j}$ and $\mathbf{M}_{j}$ are substructure contributions to the global stiffness and mass matrix, specified by the finite element model of the structure.

\subsubsection{Cost function}

As previously indicated, essential in the model updating scheme is the confrontation of the measured and computed data in a cost function $F$ (equation (2)). Many alternative formulations are possible for the cost function, but most often it is expressed as a weighted least squares fit between the predictions $\mathbf{G}_{\mathrm{M}}\left(\boldsymbol{\theta}_{\mathrm{M}}\right)$ and the data $\overline{\mathbf{d}}$ as follows:

$$
F\left(\boldsymbol{\theta}_{\mathrm{M}}\right)=\frac{1}{2} \boldsymbol{\eta}\left(\boldsymbol{\theta}_{\mathrm{M}}\right)^{\mathrm{T}} \mathbf{W} \boldsymbol{\eta}\left(\boldsymbol{\theta}_{\mathrm{M}}\right)=\frac{1}{2}\left\|\mathbf{W}^{1 / 2} \boldsymbol{\eta}\left(\boldsymbol{\theta}_{\mathrm{M}}\right)\right\|_{2}^{2}
$$

where $\|\cdot\|_{2}$ denotes the Euclidian or 2 -norm of a vector, and where $\mathbf{W} \in \mathbb{R}^{N \times N}$ is a weighting matrix. The residual vector $\boldsymbol{\eta}\left(\boldsymbol{\theta}_{\mathrm{M}}\right)=\overline{\mathbf{d}}-\mathbf{G}_{\mathrm{M}}\left(\boldsymbol{\theta}_{\mathrm{M}}\right) \in \mathbb{R}^{N}$ contains the difference between the model predictions and the data; it is therefore often referred to as the prediction error.

In most practical applications, a diagonal weighting matrix $\mathbf{W}$ is assumed, where the weighting coefficients are chosen proportionate to the inverse measured data so that the individual residuals are in effect 
normalized:

$$
F\left(\boldsymbol{\theta}_{\mathrm{M}}\right)=\frac{1}{2} \sum_{k=1}^{N} a_{k} \frac{\left(\bar{d}_{k}-y_{k}\left(\boldsymbol{\theta}_{\mathrm{M}}\right)\right)^{2}}{\bar{d}_{k}^{2}}
$$

Factors $a_{k}$ reflect the weight appointed to the normalized residuals, and can generally be interpreted as "confidence" measures representing the accuracy of the measured data on the one hand, and regarding the capability of the model class to correctly represent the true structural behavior on the other hand. In selected cases, some information regarding e.g. the measurement accuracy is available, and weighting factors can be taken inversely proportional to observed standard deviations. In fact, this can be shown to be the most optimal choice for the weighting factors, at least for linear prediction models (Gauss-Markov theorem). However, in most cases the weighting factors are chosen based on engineering judgment and/or trial-and-error.

When several types of data or residuals are used, they are usually combined in one single objective function which is then minimized. An alternative is to apply a multi-objective optimization scheme, which allows for the minimization of multiple objective functions simultaneously, thereby avoiding the challenging task of weighting the individual (and possibly contradictory) objectives. For instance, Haralampidis et al. [34] and Christodoulou and Papadimitriou [35] define separate eigenfrequency and mode shape residuals, Jaishi and Ren 30] use the approach to incorporate both eigenfrequency and strain energy residuals, and Schlune et al. 36] combine static and dynamic measurements in multiple objectives. A different approach to the modal updating problem in structural dynamics was developed by Ladevèze and his co-workers 1720]. Here, the cost function is not defined in terms of residuals in modal data such as eigenfrequencies and mode shapes but contains an energy-based indicator, termed the Constitutive Relation Error (CRE). In this approach, a distinction is made between reliable data (e.g. position and direction of sensors) and less reliable data (e.g. constitutive relation) and model updating parameters are selected after a prior localization of errors in the model based on the local values of the CRE. In the following, the focus is on model updating methods based on cost functions as given by equation (6) that contain residuals in modal data.

\subsection{Solution and ill-posedness of the optimization problem}

The (single-objective) least squares problem associated with model updating can be constructed using equations (2) and (5) as:

$$
\boldsymbol{\theta}_{\mathrm{M}}^{*}=\underset{\boldsymbol{\theta}_{\mathrm{M}}}{\arg \min } F\left(\boldsymbol{\theta}_{\mathrm{M}}\right)=\underset{\boldsymbol{\theta}_{\mathrm{M}}}{\arg \min } \frac{1}{2}\left\|\mathbf{W}^{1 / 2}\left(\overline{\mathbf{d}}-\mathbf{G}_{\mathrm{M}}\left(\boldsymbol{\theta}_{\mathrm{M}}\right)\right)\right\|_{2}^{2}
$$

The solution $\boldsymbol{\theta}_{\mathrm{M}}^{*}$ should satisfy the stationarity condition $\nabla_{\boldsymbol{\theta}_{\mathrm{M}}} F\left(\boldsymbol{\theta}_{\mathrm{M}}^{*}\right)=\mathbf{0}$. For a linear model, i.e. $\mathbf{G}_{\mathrm{M}}\left(\boldsymbol{\theta}_{\mathrm{M}}\right)=$ $\mathbf{J} \boldsymbol{\theta}_{\mathrm{M}}$, this yields the following set of normal equations for $\boldsymbol{\theta}_{\mathrm{M}}$ :

$$
\left(\mathbf{J}^{\mathrm{T}} \mathbf{W} \mathbf{J}\right) \boldsymbol{\theta}_{\mathrm{M}}=\mathbf{J}^{\mathrm{T}} \mathbf{W} \overline{\mathbf{d}}
$$

In this equation, $\mathbf{J} \in \mathbb{R}^{N \times N_{\mathrm{M}}}$ is the Jacobian or sensitivity matrix containing the first order derivatives of the $N$ model outputs with respect to the $N_{\mathrm{M}}$ model parameters, and matrix $\left(\mathbf{J}^{\mathrm{T}} \mathbf{W} \mathbf{J}\right)$ is the Hessian matrix $\mathbf{H} \in \mathbb{R}^{N_{\mathrm{M}} \times N_{\mathrm{M}}}$. It is clear that this Hessian matrix needs to be inverted in order to solve expression (8) for $\boldsymbol{\theta}_{\mathrm{M}}$. Generally, linear least squares problems can be solved relatively straightforwardly using decomposition methods such as singular value decomposition (SVD), QR factorization or Cholesky factorization.

In order for problem (8) to have a unique solution, the Hessian should be a positive definite matrix; this is generally referred to as the second order necessary optimality condition (SONC), and is in fact equivalent to having a sensitivity matrix $\mathbf{J}$ of full column rank. Even though rank-deficiency of $\mathbf{J}$ is usually not a problem in model updating, near-rank-deficiency is extremely common. This so-called ill-conditioning of $\mathbf{J}$ causes the Hessian to become near-singular and difficult to invert accurately, which results in a solution that is very unstable with respect to small changes in the data vector $\overline{\mathbf{d}}$ and the model predictions.

When the relationship between model parameters and model outputs is nonlinear - as is most often the case - the least squares problem in equation (7) cannot be solved directly anymore, but requires an iterative 
approach. Although a multitude of efficient methods has been developed [5, 37, 38], local gradient-based iterative algorithms such as the Gauss-Newton approach are most often employed. It can be shown that these methods require the solution of a set of equations very similar to (8) in each iteration, only now the Jacobian $\mathbf{J}$ and Hessian $\mathbf{H}$ are local approximations, which can be computed numerically or analytically (see below). In other words, both in the linear and in the nonlinear case, the uniqueness and stability of the solution are determined by the conditioning of the sensitivity matrix $\mathbf{J}$.

By far the most common cause of ill-conditioning in model updating is found in the incompatibility of the prediction model and its parameterization with the resolution and type of the observational data [39]. In general, ill-conditioning through parameterization can be avoided or abated by (1) choosing physically relevant parameters that sufficiently affect the observed data (thereby avoiding near-zero columns in the sensitivity matrix); and (2) avoiding overparameterization along the structure (thereby avoiding nearly linearly dependent columns in the sensitivity matrix for neighboring elements). The latter is usually accomplished by not defining the updating parameter element-wise, but instead for sets of (adjacent) elements, also referred to as substructures. Alternatively, the variation of the parameter values along the structure can be described by a function (e.g. a linear or quadratic interpolation function) which is characterized by only a few parameters, thus reducing the number of updating parameters significantly [40]. Additionally, it is often advantageous to select correction factors instead of the physical parameters themselves; as such it is avoided that largely varying orders of magnitude cause numerical difficulties (inaccuracy) when inverting the Hessian matrix.

When ill-conditioning cannot be resolved by well thought out parameterization of the model, regularization techniques may provide an answer. The basic idea behind these methods is to replace the ill-posed problem with a well-conditioned one that behaves similarly and produces an approximate solution to the original problem. Usually, this is done by augmenting the objective function with a regularization term that incorporates some form of prior information on the solution (e.g. smoothness, sparsity, etc). For further details regarding regularization techniques, the reader is referred to the extensive literature by Hansen [41] and Neumaier 42]. A detailed review of regularization techniques in model updating can be found in Titurus and Friswell 43 .

\section{4. $R C$ beam example}

Experimental data. For the RC beam introduced in section 1.3. a set of four experimental natural frequencies and mode shapes are extracted from the time-domain vibration data by stochastic subspace identification (SSI) [44]. The transverse mode shape displacements are observed at $N_{o}=31$ equidistant locations along the beam; the resulting mode shape measurements are shown with their corresponding natural frequencies $\bar{f}_{r}$ in figure 3

Prediction model. A 2D FE beam model of the reinforced concrete beam is constructed which consists of 30 elements and 31 nodes, with two DOFs per node so that $N_{d}=62$. Shear deformation is included in the FE model, with a factor $k_{y}=A / A_{s}=1.2$, and the moment of inertia of the equivalent cross-section equals $1.93 \times 10^{-4} \mathrm{~m}^{4}$. Substructuring is applied in order to avoid ill-conditioning: the beam is divided into 10 substructures with a uniform stiffness value or Young's modulus (figure 4); these 10 stiffness values are adopted as the updating parameters $\boldsymbol{\theta}_{\mathrm{M}}$. Initially, all stiffness parameters are assumed to equal $\theta_{\mathrm{M} 0}=37.5$ GPa.

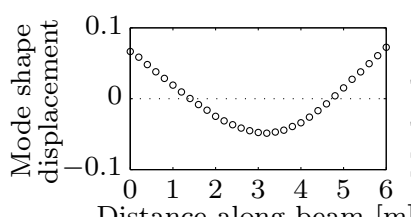

Distance along beam $[\mathrm{m}]$

(a) $\bar{f}_{1}=19.49 \mathrm{~Hz}$

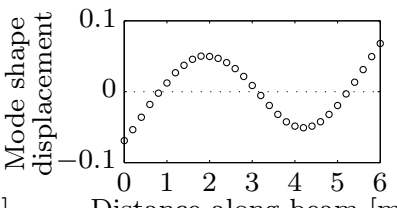

Distance along beam $[\mathrm{m}]$

(b) $\bar{f}_{2}=56.66 \mathrm{~Hz}$

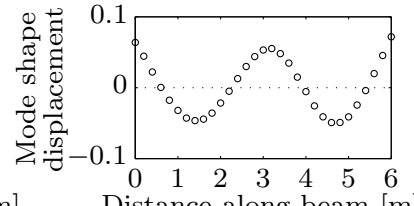

(c) $\bar{f}_{3}=111.2 \mathrm{~Hz}$

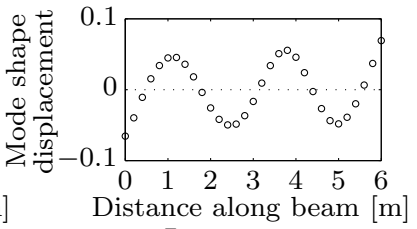

(d) $\bar{f}_{4}=184.9 \mathrm{~Hz}$

Figure 3: First four experimental bending modes and corresponding natural frequencies for the damaged state. 


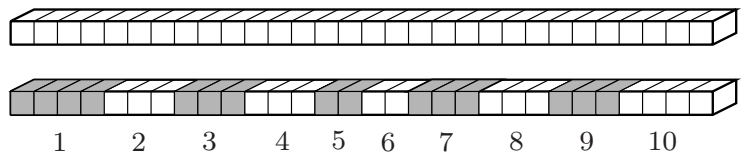

Figure 4: Definition of the 10 substructures in the RC beam.

Cost function. In view of the employed modal data, the prediction error can be partitioned as $\boldsymbol{\eta}=\left\{\boldsymbol{\eta}_{\lambda} ; \boldsymbol{\eta}_{\phi}\right\}$, where the eigenvalue and mode shape residual vectors are defined as $\eta_{\lambda}=\left\{\ldots ; \eta_{\lambda, r} ; \ldots\right\} \in \mathbb{R}^{N_{m}}$ and $\boldsymbol{\eta}_{\phi}=\left\{\ldots ; \boldsymbol{\eta}_{\phi, r} ; \ldots\right\} \in \mathbb{R}^{N_{m} N_{o}}$, respectively. In the present work, it is chosen to define the individual modal residuals as follows:

$$
\begin{array}{lll}
\eta_{\lambda, r}=\bar{\lambda}_{r}-\lambda_{r}\left(\boldsymbol{\theta}_{\mathrm{M}}\right) & \in \mathbb{R} \\
\boldsymbol{\eta}_{\phi, r}=\gamma_{r} \overline{\boldsymbol{\phi}}_{r}-\mathbf{L} \boldsymbol{\phi}_{r}\left(\boldsymbol{\theta}_{\mathrm{M}}\right) & \in \mathbb{R}^{N_{o}}
\end{array}
$$

where $r=1 \ldots N_{m}$, and the binary matrix $\mathbf{L} \in \mathbb{R}^{N_{o} \times N_{d}}$ selects the $N_{o}$ observed degrees of freedom from the $N_{d}$ degrees of freedom present in the FE model. The coefficient $\gamma_{r}$ is a scaling factor to ensure that simulated and measured mode shapes are scaled equally. This is necessary since in most cases, the experimental modes are identified using output-only data, and therefore cannot be mass-normalized. The scaling factor can be obtained from a reference value at a single observation point or, alternatively, through a least squares fit between measured and computed mode shapes, which yields:

$$
\gamma_{r}=\frac{\bar{\phi}_{r}^{\mathrm{T}} \mathbf{L} \phi_{r}\left(\boldsymbol{\theta}_{\mathrm{M}}\right)}{\left\|\bar{\phi}_{r}\right\|_{2}^{2}}
$$

The construction of the cost function further requires a matching of the $N_{d}$ calculated modes to the $N_{m}$ experimental mode shapes, in order to ensure that corresponding modes are compared. To this end, the modal assurance criterion (MAC) is frequently applied, which consists of computing so-called MAC-values as a measure for the correspondence between a calculated mode shape $\phi_{i}$ and a measured mode shape $\bar{\phi}_{j}$ :

$$
\operatorname{MAC}\left(\phi_{i}, \bar{\phi}_{j}\right)=\frac{\left|\left(\mathbf{L} \phi_{i}\right)^{\mathrm{T}} \bar{\phi}_{j}\right|^{2}}{\left\|\mathbf{L} \phi_{i}\right\|_{2}^{2}\left\|\bar{\phi}_{j}\right\|_{2}^{2}}
$$

The MAC-values always lie between 0 and 1 , where values closer to 1 represent a better agreement between the considered mode shapes. In selected cases with many closely spaced or very similar modes, the MACapproach may lead to erroneous mode matching; therefore, alternative mode matching methods often include an eigenfrequency or eigenvalue residual as well, for instance by matching modes that result in the lowest value of the following quantity:

$$
1-\operatorname{MAC}\left(\phi_{i}, \bar{\phi}_{j}\right)+\left|1-\frac{\lambda_{i}}{\bar{\lambda}_{j}}\right|
$$

It is important to note that mode shape matching often results in non-smooth behavior of the objective function. As the parameter values vary, the mode shapes might get matched differently, resulting in sudden jumps in the objective function which can hinder the efficiency of gradient-based optimization algorithms significantly.

A normalized least squares cost function as defined in equation (6) is adopted, which in this case leads to the following reformulation of the objective function:

$$
F\left(\boldsymbol{\theta}_{\mathrm{M}}\right)=\frac{1}{2} \sum_{r=1}^{N_{m}} a_{r} \frac{\left(\bar{\lambda}_{r}-\lambda_{r}\left(\boldsymbol{\theta}_{\mathrm{M}}\right)\right)^{2}}{\bar{\lambda}_{r}^{2}}+\frac{1}{2} \sum_{r=1}^{N_{m}} b_{r} \frac{\left\|\gamma_{r} \overline{\boldsymbol{\phi}}_{r}-\mathbf{L} \boldsymbol{\phi}_{r}\left(\boldsymbol{\theta}_{\mathrm{M}}\right)\right\|_{2}^{2}}{\left\|\gamma_{r} \overline{\boldsymbol{\phi}}_{r}\right\|_{2}^{2}}
$$


The computed and measured mode shapes are matched and scaled through the MAC and least squares scaling, respectively. The weighting factors $a_{r}$ and $b_{r}$ in the cost function are taken equal to one to obtain equal weighting between the terms containing eigenvalue and mode shape residuals. The model updating procedure for the RC beam now consists in minimizing objective function (14) with respect to $\boldsymbol{\theta}_{\mathrm{M}}$. This can be achieved through any optimization software; in this case, the nonlinear least squares optimization tool (lsqnonlin) in MATLAB [45] is employed, which here results in the application of the Levenberg-Marquardt variant of the Gauss-Newton algorithm. The initial model is used as starting point for the algorithm.

Modal sensitivities. Most gradient-based optimization methods require the computation of the first and second order sensitivities of the model predictions with respect to the model parameters, which are collected in the Jacobian and Hessian matrix, respectively. To this end, two basic approaches are available. By far the most popular is making use of the finite difference method; however, this approach is very time-consuming and only locally accurate. Alternatively, use can be made of analytical expressions.

In vibration-based model updating, the required sensitivities relate to the predicted eigenvalues and mode shapes. The eigenfrequency derivatives can be readily computed from the undamped eigenvalue equation $\mathbf{K} \phi_{r}-\lambda_{r} \mathbf{M} \phi_{r}=0$, yielding:

$$
\begin{aligned}
\frac{\partial \lambda_{r}}{\partial \theta_{i}} & =\boldsymbol{\phi}_{r}^{\mathrm{T}} \frac{\partial \mathbf{K}}{\partial \theta_{i}} \boldsymbol{\phi}_{r} \\
\frac{\partial^{2} \lambda_{r}}{\partial \theta_{i} \partial \theta_{j}} & =\left(\frac{\partial \phi_{r}}{\partial \theta_{j}}\right)^{\mathrm{T}} \frac{\partial \mathbf{K}}{\partial \theta_{i}} \boldsymbol{\phi}_{r}+\boldsymbol{\phi}_{r}^{\mathrm{T}} \frac{\partial \mathbf{K}}{\partial \theta_{i}} \frac{\partial \boldsymbol{\phi}_{r}}{\partial \theta_{j}}
\end{aligned}
$$

for the common case where $\mathbf{M}\left(\boldsymbol{\theta}_{\mathrm{M}}\right)=\mathbf{M}$. Second order eigenvalue derivatives for the case where the mass matrix is dependent on the model parameters can be found e.g. in Ghosh et al. [46].

Several approaches exist to compute the first and second order derivatives of the modal vectors. The most commonly used method is based on the work of Fox and Kapoor [47], where eigenvector sensitivities are described as a linear combination of all eigenvectors. In order to obtain exact derivatives, this straightforward approach requires the solution of the $\left(N_{d} \times N_{d}\right)$ FEM eigenproblem for each considered parameter set, which can quickly become computationally prohibitive for large systems with many degrees of freedom $N_{d}$, as often encountered in real (large-scale) applications. In practice the summation is therefore often truncated to the $N_{d}^{*}$ lowest modes, yielding an approximation of the derivatives. A suitable truncation base $N_{d}^{*}$ is usually determined by trial-and-error.

Nelson [48] developed an alternative, exact approach for computing the eigenvector derivatives which only requires the computation of the modal data of the $N_{m}$ modes of interest, and the solution of a $\left(N_{d} \times N_{d}\right)$ linear system of equations for each set of parameter values. Further details on computing first and second order mode shape derivatives using Nelson's method can be retrieved e.g. in [49].

Results. From table 1] it is clear that the updated natural frequencies show a much better correspondence with the experimental results compared to the initial predictions, especially for modes 2 and 4. The MACvalues after updating confirm that a good fit is obtained for the mode shapes as well. The updated bending stiffness values along the beam are shown in figure 5. where the damage induced by the static load at about $4 \mathrm{~m}$ is clearly reflected by a decrease of the bending stiffness by about $50 \%$ at this location. This application shows how a relatively limited amount of modal data and a simple FE prediction model can help identifying, locating, and quantifying structural damage. The question that now arises is how measurement 
and modeling uncertainty affect the updating results.

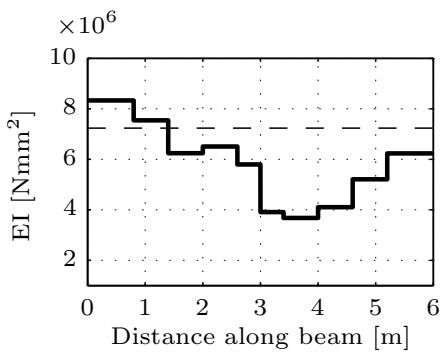

Figure 5: Updated bending stiffness pattern of the RC beam.

\begin{tabular}{c|c|ccc|ccc}
\hline \hline Mode & $\begin{array}{c}f_{\text {init }} \\
{[\mathrm{Hz}]}\end{array}$ & $\begin{array}{c}f_{\text {exp }} \\
{[\mathrm{Hz}]}\end{array}$ & $\begin{array}{c}\Delta f_{\text {init }} \\
{[\%]}\end{array}$ & $\begin{array}{c}\text { MAC }_{\text {init }} \\
{[-]}\end{array}$ & $\begin{array}{c}f_{\text {upd }} \\
{[\mathrm{Hz}]}\end{array}$ & $\begin{array}{c}\Delta f_{\text {upd }} \\
{[\%]}\end{array}$ & $\begin{array}{c}\mathrm{MAC}_{\text {upd }} \\
{[-]}\end{array}$ \\
\hline 1 & 24.04 & 19.49 & 23.35 & 0.991 & 19.58 & 0.49 & 0.997 \\
2 & 65.79 & 56.66 & 16.11 & 0.989 & 56.65 & 0.02 & 0.999 \\
3 & 127.6 & 111.2 & 14.74 & 0.976 & 110.8 & 0.36 & 0.999 \\
4 & 208.2 & 184.9 & 12.56 & 0.964 & 184.7 & 0.13 & 0.999 \\
\hline \hline
\end{tabular}

Table 1: Natural frequencies $f_{\text {init }}$ calculated with the initial FE model, the experimental frequencies $f_{\exp }$ and relative frequency differences $\Delta f=$ $\left|f_{\exp }-f\right| / f_{\exp }$ and MAC-values.

\section{Uncertainty in model updating}

The above discourse in section 2.3 regarding the ill-posedness of the inverse problem demonstrates that model updating is affected by uniqueness and stability issues, and is therefore extremely susceptible to errors, or, in more general terminology, to uncertainties. Effectively and comprehensively accounting for these uncertainties to assess their influence is therefore an extremely valuable if not indispensable undertaking in model updating. Moreover, uncertainty quantification is instrumental in exploring and improving the robustness of the problem and derived applications.

Uncertainty is inherently present in all of reality, and is most generally defined by Walker et al. [50] as "any departure from the unachievable ideal of complete determinism". Over the last decades, a wide array of different classification systems and analysis methods has been developed, originating in the field of risk analysis and extending to practically all engineering and science fields, mainly in an effort to manage uncertainty and its consequences in a comprehensive fashion. In this section, an overview is presented of the different types and sources of uncertainties in model updating, classifying them first by context.

\subsection{Uncertainty related to the prediction model}

Every numerical prediction model is, by definition, an idealized representation of reality and therefore inherently unable to perfectly represent the behavior of the true system. Many authors such as Mottershead and Friswell [1], Der Kiureghian [51], Soize [52, 53], Kennedy and O'Hagan [54] and Walker [50] distinguish several types or sources of model uncertainty, which can be summarized as follows:

- Model parameter uncertainty, also referred to as model input uncertainty or variable uncertainty. This type of uncertainty is caused by uncertainty regarding the values of parameters or inputs of the selected prediction model. The model parameters can be related to unknown or insufficiently known material properties, load characteristics or geometric properties, but can also represent more "global" parameters, that e.g. determine features of the structure of the model.

- Model structure uncertainty, also referred to as model framework or model form uncertainty. This kind of uncertainty arises as a result of modeling assumptions and simplifications made by the designer in answer to a lack of knowledge or understanding of the true system. Typical examples of model uncertainty are uncertain model context (i.e. uncertain extent of the numerical model) and assumptions of unknown validity regarding the selected model parameters, boundary conditions, governing physical equations and model order. Legault et al. [55] make a distinction between deliberate simplifications made by the modeler (e.g. based on the desired complexity and fidelity of the model) and unintended simplifications, resulting from a lack of understanding of the system. Uncertainties related to the mathematical algorithms used to compute the output, such as discretization error (e.g. for FE models) and approximation error (e.g. when sampling or simulation methods are applied) can be interpreted as model structure error as well. 
- Model code uncertainty, also referred to as numerical uncertainty or technical model uncertainty, is usually ignored as it is typically assumed to only account for a negligible part of the modeling error. It is described as the uncertainty that results from errors in the computer implementation (i.e. software or hardware errors). Kennedy and O'Hagan [54] and O'Hagan and Oakley [56] argue that approximation error should be categorized as model code uncertainty, as it results from an effort to numerically approximate the model output after the model structure has been decided upon. Most often, however, approximation error is classified under model structure uncertainty, and model code uncertainty is assumed negligible.

Due to model uncertainty, a discrepancy arises between the model predictions $\mathbf{G}_{\mathrm{M}}\left(\boldsymbol{\theta}_{\mathrm{M}}\right)$ and the true system output $\mathbf{d}$; this difference is defined as the modeling error $\boldsymbol{\eta}_{\mathrm{G}}$ :

$$
\boldsymbol{\eta}_{\mathrm{G}}=\mathbf{d}-\mathbf{G}_{\mathrm{M}}\left(\boldsymbol{\theta}_{\mathrm{M}}\right) \quad \text { or } \quad \mathbf{d}=\mathbf{G}_{\mathrm{M}}\left(\boldsymbol{\theta}_{\mathrm{M}}\right)+\boldsymbol{\eta}_{\mathrm{G}}
$$

The presence of modeling error implies that the structural model class never contains the true structural system.

\subsection{Uncertainty related to the experimental data}

Since model updating makes use of observational data, the most obvious source of uncertainty relates to measurement or experimental error. This error can be random measurement noise, or it can be a bias or systematic error caused by imperfections in the measurement equipment or setup, or during the subsequent signal processing. Measurement error forms a source of discrepancy between the observed structural behavior $\overline{\mathbf{d}}$ and the real structure response $\mathbf{d}$; in the following, this difference is defined as the measurement error $\boldsymbol{\eta}_{\mathrm{D}}$ :

$$
\eta_{\mathrm{D}}=\overline{\mathbf{d}}-\mathbf{d} \quad \text { or } \quad \overline{\mathbf{d}}=\mathbf{d}+\boldsymbol{\eta}_{\mathrm{D}}
$$

In FE model updating, the experimental data (i.e. modal characteristics) are extracted from time-domain vibration data using system identification methods [22]. In these methods, a linear time-invariant (LTI) system is selected to approximate the true system, which is then employed to identify the modal characteristics. Besides measurement noise, a number of factors can result in uncertainty on these extracted modal data, e.g. the finite nature of the data, filtering of the data, the selected model order and the chosen LTI model structure. It is clear that the measurement error is in this case partly caused by a form of model (structure) uncertainty regarding the chosen LTI system. Typically these uncertainties give rise to a bias of the model (resulting e.g. in identification of spurious modes), bias of the modes, and variance errors. In most situations, making use of stabilization diagrams and careful selection of the model order can resolve the bias errors, whereas the variance error on the modes cannot be reduced. It can, however, be estimated, e.g. using the method developed by Reynders et al. [57].

\subsection{Combining measurement and model uncertainty}

Eliminating the (unknown) true system output $\mathbf{d}$ from equations (17) and (18) results in:

$$
\mathrm{G}_{\mathrm{M}}\left(\boldsymbol{\theta}_{\mathrm{M}}\right)+\boldsymbol{\eta}_{\mathrm{G}}=\overline{\mathbf{d}}-\boldsymbol{\eta}_{\mathrm{D}}
$$

This equation illustrates that the model prediction corrected by the modeling error $\boldsymbol{\eta}_{\mathrm{G}}$ is equal to the numerical observations corrected by the measurement error $\boldsymbol{\eta}_{\mathrm{D}}$. Therefore, each side of the equation forms an expression for the true unknown output of the system. When both errors are collected on the right hand side of this equation, the total observed prediction error $\boldsymbol{\eta}$ is obtained as the difference between the model predictions and the observed quantities:

$$
\overline{\mathbf{d}}-\mathbf{G}_{\mathrm{M}}\left(\boldsymbol{\theta}_{\mathrm{M}}\right)=\boldsymbol{\eta}_{\mathrm{G}}+\boldsymbol{\eta}_{\mathrm{D}}=\boldsymbol{\eta}
$$

Due to the presence of measurement and modeling error, the true system behavior and predictions made by the model are uncertain, meaning that the results of a model updating procedure are by definition also 
uncertain. In order to quantify and account for these uncertainties, a number of different approaches may be applied; the above expression (20) serves as a starting point for all uncertainty quantification approaches for model updating.

\subsection{Modeling of uncertainties}

Methods aimed at uncertainty quantification can generally be classified into two main categories: probabilistic and non-probabilistic approaches. The first category represents the classical approach for uncertainty modeling, set on the firm foundations of probability theory, where uncertainty is modeled by appointing probability density functions (PDFs) to uncertain quantities; these PDFs are then propagated to probabilistic descriptions of the outputs of interest. These so-called parametric approaches are well-established in structural and mechanical engineering; comprehensive reviews on stochastic mechanics can be found in the works of Schuëller et al. [58 61] and special issues of Computer Methods in Applied Mechanics and Engineering [62], Computers and Structures [63] and Probabilistic Engineering Mechanics [64]. One of the most widely used approaches in mechanics is the stochastic finite element method (SFEM) 65, 66]. More recently, the so-called non-parametric approach was developed by Soize 67, 68]. This methods makes use of random matrix theory [69] to construct a probabilistic model of the prediction model operator $\mathbf{G}_{\mathrm{M}}$. Although Legault et al. [55] describe some reservations regarding the physical implications of the method, the nonparametric approach has a wide application domain [70].

Specifically for uncertainty quantification in inverse problems, many alternative probabilistic strategies are available. Here, a distinction can be made between classical methods and Bayesian methods. The classical methods are based on estimating some statistics of the parameters (e.g. mean values and/or covariance matrices) so that the statistics of the output of the model correspond in some optimal way to the statistics of the observed data. This can be done analytically (i.e. classical statistical inference methods 71, 72], recent applications in 73,74$]$ ) or numerically (i.e. stochastic model inversion, applications in 75 78]). Methods characterizing the parameter uncertainty by the mean values and covariance matrices have as advantage that these low-order statistics can generally be computed more easily and at a lower computational cost than in the case where a full probabilistic model is considered. An alternative classical approach consists in the deterministic inversion of a large number of data points, and constructing a suitable probabilistic model of the parameters (recent applications in e.g. 81 84]). It is clear that this approach requires a large amount of repeated measurements, and in most cases only allows incorporating variability in the observations.

The Bayesian inference approach was introduced in structural dynamics by Beck et al. [85], and complements the probabilistic model description with a probabilistic model for the prediction error $\boldsymbol{\eta}$; this model then represents both the measurement and modeling uncertainty. Using the well-known Bayes' theorem, prior probabilistic models, which are constructed based on the a priori available information, can be transformed into posterior models, using the available experimental data and the probabilistic prediction error model. The Bayesian approach is particularly suited for inverse problems, and will constitute the main topic of section 4 .

The last decades saw a surge in non-probabilistic methods for uncertainty modeling, in response to some apparent drawbacks/limitations of the probabilistic method related to the construction of the PDFs and modeling of epistemic uncertainty. Most well-known are the interval analysis approach, convex modeling and fuzzy set theory; this latter approach is discussed in detail in section 5. Interesting applications of the interval approach in structural mechanics and dynamics can be found in Rao and Berke [86], Muhanna and Mullen [87] and Moens et al. [88]. In recent years, the interval approach has also been applied for FE model updating, see e.g. Gabriele and Valente [89], García et al. 90] and Khodaparast et al. [91, 92]. The interval method can be seen as a specific case of the more general convex modeling approach, coined by Ben-Haim and Elishakoff [93], where it is assumed that the uncertain quantities lie within a convex region. Applications of this approach in structural engineering can be found e.g. in [94, 95].

Besides pure non-probabilistic methods, a class of mixed or hybrid approaches evolved as well. The most commonly used methods include probability bound analysis 96. (also referred to as p-box approach, method of lower and upper previsions [97] or interval probabilities [98]), random set theory [99, 100] (also referred to as evidence theory), and fuzzy randomness [101 104]. Mixed methods are not treated in the present work. 


\section{Bayesian FE model updating}

Bayesian methods are founded on the well-known Bayes' theorem, introduced in the 18th century by Thomas Bayes [105] and generalized later by Pierre-Simon Laplace [106] into its currently known form. The theorem expresses conditional probability, or, very generally, the probability of statement $A$ given statement $B$. Depending on the employed interpretation of probability, the meaning of Bayes' theorem differs significantly. In the so-called frequentist interpretation pertained in classical statistics, probability is interpreted as a measure for the (long-term) frequency of the occurrence of a certain event. Then, Bayes' theorem expresses the proportion of events $A$ that occur, given the occurrence of another event $B$. In Bayesian statistics, on the other hand, probability is assumed to reflect the relative plausibility or degree of belief attributed to a certain event, hypothesis or proposition, given the current state of information. In this interpretation, the Bayes' theorem forms a mathematical basis for adjusting or updating the probability of a certain hypothesis $(A)$ as more evidence or data $(B)$ on the hypothesis becomes available - this form of statistical inference is referred to as Bayesian inference. It is clear that only this Bayesian interpretation - often also termed the Cox-Jaynes interpretation [107, 108] - is meaningful in the interest of forming inferences regarding e.g. model parameters based on observed data. Moreover, the Bayesian interpretation automatically provides a rigorous framework for uncertainty quantification: the degree of belief attributed to a certain quantity in effect reflects the uncertainty that is believed to be associated with that quantity. Note that this interpretation also avoids the need for the classical distinction between aleatory and epistemic uncertainty [51]; in fact, all uncertainties are presumed as epistemic [56].

Due to the method's extensive application field and long history, a vast amount of literature is available on Bayesian inference; in the last few decades, important reference works in the context of model updating were developed by Beck and Katafygiotis [85, 109], Katafygiotis et al. [110], Beck [111], Jaynes [108], Mosegaard and Tarantola 112, 113], Box and Tiao 114, Peterka 115] and Kennedy and O'Hagan 54].

One of the most popular uses of Bayesian inference in civil and mechanical engineering is structural health monitoring (SHM), where Beck and co-workers may form the most well-represented group in terms of reference publications. Vanik et al. [116] applied the method set out by Beck and Katafygiotis [85] for SHM purposes in 2000, and showed that the Bayesian approach allows to obtain updated probabilities of model parameters as well as probabilistic damage measures, based on observations of structural behavior. In the following years, Beck et al. 117, Yuen et al. 118 120], Ching et al. 121 123, and Cheung et al. 124. employed this approach in many applications, demonstrating and extending the method's applicability and efficiency for many types of structural (finite element) models. Papadimitriou et al. [125] and Choi et al. 126] used Bayesian inference to obtain structural reliability updates; Sohn and Law [127, 128] used the approach to compute probabilities of different damage scenarios.

The present work will elaborate the specifics of the application of Bayesian inference for uncertainty quantification in finite element model updating.

\subsection{Probabilistic uncertainty modeling}

As mentioned above, the basic principle behind the Bayesian method is that, besides the uncertain model parameters $\boldsymbol{\theta}_{\mathrm{M}} \in \mathbb{R}^{N_{\mathrm{M}}}$, the measurement and modeling errors are modeled as random variables as well. PDFs are appointed to $\boldsymbol{\eta}_{\mathrm{G}}$ and $\boldsymbol{\eta}_{\mathrm{D}}$, which are parameterized by parameters $\boldsymbol{\theta}_{\mathrm{G}} \in \mathbb{R}^{N_{\mathrm{G}}}$ and $\boldsymbol{\theta}_{\mathrm{D}} \in \mathbb{R}^{N_{\mathrm{D}}}$. These parameters are added to the structural model parameters $\boldsymbol{\theta}_{\mathrm{M}}$ to form the general model parameter set $\boldsymbol{\theta}=\left\{\boldsymbol{\theta}_{\mathrm{M}} ; \boldsymbol{\theta}_{\mathrm{G}} ; \boldsymbol{\theta}_{\mathrm{D}}\right\} \in D \subseteq \mathbb{R}^{N_{\theta}}$. This in fact corresponds to adding two probabilistic model classes to the structural model class $\mathcal{M}_{\mathrm{M}}$ to form a joint model class $\mathcal{M}=\mathcal{M}_{\mathrm{M}} \times \mathcal{M}_{\mathrm{G}} \times \mathcal{M}_{\mathrm{D}}$, parameterized by $\boldsymbol{\theta}$. This procedure of probabilistic modeling is commonly referred to as stochastic embedding [111, 129].

\subsection{Bayes' theorem for model updating}

Bayesian inference allows for the updating of the probabilities of the unknown parameters $\boldsymbol{\theta}$ characterizing a certain joint model class $\mathcal{M}$ when some observations $\overline{\mathbf{d}}$ become available. This is done through the wellknown Bayes' theorem of conditional probability:

$$
p(\boldsymbol{\theta} \mid \overline{\mathbf{d}}, \mathcal{M})=c p(\overline{\mathbf{d}} \mid \boldsymbol{\theta}, \mathcal{M}) p(\boldsymbol{\theta} \mid \mathcal{M})
$$


In this equation, $p(\boldsymbol{\theta} \mid \overline{\mathbf{d}}, \mathcal{M})$ is the updated or posterior PDF of the model parameters given the measured data $\overline{\mathbf{d}}$ and the assumed model class $\mathcal{M} ; p(\overline{\mathbf{d}} \mid \boldsymbol{\theta}, \mathcal{M})$ is the PDF of the observed data given the parameters $\boldsymbol{\theta}$; and $p(\boldsymbol{\theta} \mid \mathcal{M})$ is the initial or prior PDF of the parameters. In the following, the explicit dependence on the model class $\mathcal{M}$ is omitted in order to simplify the notations. The normalizing constant $c$ ensures that the posterior PDF integrates to one, so that:

$$
c^{-1}=\int_{D} p(\overline{\mathbf{d}} \mid \boldsymbol{\theta}) p(\boldsymbol{\theta}) d \boldsymbol{\theta}
$$

This integral is equal to $p(\overline{\mathbf{d}} \mid \mathcal{M})$, which is often referred to as the evidence for model class $\mathcal{M}$ and plays a determining role in Bayesian model class selection [130, 131].

\subsection{The prior $P D F$}

The prior PDF $p(\boldsymbol{\theta})$ reflects the probability of model parameters $\boldsymbol{\theta}$ in the absence of observed information; it is obtained independently of current measurement results. In many cases, this PDF is chosen based on engineering judgment. A common criticism that arises in this context is the problem of arbitrariness or subjectivity, i.e. there is no guarantee that two observers with the same prior information will conclude to the same prior PDF. This is very relevant since the prior PDF can have a significant influence on the results of the Bayesian scheme, especially in applications where the data set is small or not very informative. In situations where a large amount of informative observations is at hand, the choice of the prior PDF is not extremely determinative, since almost any prior which is smooth in the region of high likelihood (see below) will lead to similar final conclusions.

Over the last few decades, a multitude of methods aimed at obtaining theoretically consistent prior PDFs was developed; interesting review papers can be found in the works of Jaynes [132], Bernardo [133] and Berger [134]. A well-established approach is that of conjugate priors [135], where the prior PDF is chosen (based on the likelihood function) in such a way that the posterior PDF belongs to the same family of distributions. For instance, adopting a Gaussian prior when the likelihood is Gaussian will result in a Gaussian posterior PDF; it is said that the family of Gaussian distributions is self-conjugate. The main advantage of this approach is that it facilitates the exact computation of complete posterior distributions substantially, a non-negligible issue in the pre-computer era. The conjugate prior approach remains relevant, however, as it allows for improved insight into how exactly the data alter or update the prior PDF. Other approaches start from the premise that the prior PDF should add as little information as possible to the available prior information. Early methods were mainly based on invariance requirements, which led e.g. to the commonly used Jeffreys' prior [136], that assigns a uniform prior distribution to the logarithm of continuous positive variables. The last few decades saw a rise in more formal definitions of avoiding adding information through the prior, mainly based on information-theoretical criteria. For instance, Bernardo [133, 134 introduced the concept of reference priors, which aim to maximize the expected Kullback-Leibler divergence of the posterior PDF with respect to the prior.

The most widely used method, however, chooses the prior PDF based on the principle of maximum entropy, introduced for Bayesian inference by Jaynes in 1957 [137, 138] and elaborated in recent years by Gull [139] and Soize [140, 141]. The maximum entropy (ME) principle expresses that the probability distribution which best represents the current state of knowledge on some parameters $\boldsymbol{\theta}$ is the distribution which results in the largest information entropy $h_{\mathrm{pr}}$. It can be shown that a certain state of information will correspond to a single maximum entropy distribution. The ME procedure leads e.g. to a uniform prior $\mathrm{PDF}$ when it is known that a parameter is non-zero over a finite range of values in a certain interval. When the a priori available information consists of a finite mean value and finite variance of $(\log ) \theta$, it can be shown that the ME principle leads to a (log-)normal distribution. For multivariate cases, the maximum entropy principle always leads to independent prior variables, which means the joint prior PDF of $\boldsymbol{\theta}$ is constructed as the product of the marginal prior PDFs of the individual parameters $\theta_{j}$. In structural dynamics applications, the parameters $\theta_{j}$ often concern stiffness values, which are a priori known to have a positive support; furthermore, in order to ensure that the response attains finite variance, $\theta_{j}$ and $1 / \theta_{j}$ 
should in this case be second order variables 142]. It can be shown that given this prior information, the ME principle yields a gamma distribution.

\subsection{The likelihood function}

The PDF of the experimental data $p(\overline{\mathbf{d}} \mid \boldsymbol{\theta})$ can be interpreted as a measure of how good a model succeeds in explaining the observations $\overline{\mathbf{d}}$. As this PDF reflects the likelihood of observing the data $\overline{\mathbf{d}}$ when the model is parameterized by $\boldsymbol{\theta}$, it is also referred to as the likelihood function $L(\boldsymbol{\theta} \mid \overline{\mathbf{d}})$. Since the data set $\overline{\mathbf{d}}$ is fixed, this function in fact no longer represents a conditional PDF, and can be denoted as $L(\boldsymbol{\theta} ; \overline{\mathbf{d}})$; in the following, however, the common notation of $L(\boldsymbol{\theta} \mid \overline{\mathbf{d}})$ is retained. The likelihood function can be determined according to the law of total probability and equations (17) and (18) using the probabilistic models of the measurement and modeling errors:

$$
\begin{aligned}
p(\overline{\mathbf{d}} \mid \boldsymbol{\theta}) \equiv L(\boldsymbol{\theta} \mid \overline{\mathbf{d}}) & =\int_{\mathbb{R}^{N}} p_{\overline{\mathbf{d}}}(\overline{\mathbf{d}} \mid \boldsymbol{\theta}, \mathbf{d}) p_{\mathbf{d}}(\mathbf{d} \mid \boldsymbol{\theta}) d \mathbf{d} \\
& =\int_{\mathbb{R}^{N}} p_{\boldsymbol{\eta}_{\mathrm{D}}}\left(\overline{\mathbf{d}}-\mathbf{d} \mid \boldsymbol{\theta}_{\mathrm{D}}\right) p_{\mathbf{d}}(\mathbf{d} \mid \boldsymbol{\theta}) d \mathbf{d} \\
& =\int_{\mathbb{R}^{N}} p_{\boldsymbol{\eta}_{\mathrm{D}}}\left(\overline{\mathbf{d}}-\mathbf{d} \mid \boldsymbol{\theta}_{\mathrm{D}}\right) p_{\boldsymbol{\eta}_{\mathrm{G}}}\left(\mathbf{d}-\mathbf{G}_{\mathrm{M}}\left(\boldsymbol{\theta}_{\mathrm{M}}\right) \mid \boldsymbol{\theta}_{\mathrm{G}}\right) d \mathbf{d}
\end{aligned}
$$

where $p_{\boldsymbol{\eta}_{\mathrm{D}}}\left(\overline{\mathbf{d}}-\mathbf{d} \mid \boldsymbol{\theta}_{\mathrm{D}}\right)$ corresponds to the probability of obtaining a measurement error $\boldsymbol{\eta}_{\mathrm{D}}$, given the PDF of $\boldsymbol{\eta}_{\mathrm{D}}$ parameterized by $\boldsymbol{\theta}_{\mathrm{D}}$, and where $p_{\boldsymbol{\eta}_{\mathrm{G}}}\left(\mathbf{d}-\mathbf{G}_{\mathrm{M}}\left(\boldsymbol{\theta}_{\mathrm{M}}\right) \mid \boldsymbol{\theta}_{\mathrm{G}}\right)$ represents the probability of obtaining a modeling error $\boldsymbol{\eta}_{\mathrm{G}}$ when the PDF of $\boldsymbol{\eta}_{\mathrm{G}}$ is known and parameterized by $\boldsymbol{\theta}_{\mathrm{G}}$. In this derivation, it is implicitly assumed that the modeling error and measurement error are independent variables.

The above equation (24) shows that the likelihood function can be computed as the convolution of the PDFs of the measurement and modeling error. When no information is available on the individual errors, the likelihood function can be constructed using the probabilistic model of the total prediction error $\boldsymbol{\eta}$, parameterized by $\boldsymbol{\theta}_{\eta}$ :

$$
p(\overline{\mathbf{d}} \mid \boldsymbol{\theta}) \equiv L(\boldsymbol{\theta} \mid \overline{\mathbf{d}})=p\left(\overline{\mathbf{d}}-\mathbf{G}_{\mathrm{M}}\left(\boldsymbol{\theta}_{\mathrm{M}}\right) \mid \boldsymbol{\theta}\right)=p\left(\boldsymbol{\eta} \mid \boldsymbol{\theta}_{\eta}\right)
$$

Contrary to the prior PDF, the construction of the likelihood function has received much less attention in recent research, even though it has a significant influence on the Bayesian updating results. This is mainly due to the fact that most often very little or no information is at hand regarding the characteristics of the error(s); only in selected cases, a realistic estimate can be made concerning the probabilistic model representing the prediction error, for instance based on the analysis of measurement results. Usually, it is simply assumed that the probabilistic model of the prediction error is known and fixed, so that the parameter set reduces to $\boldsymbol{\theta}=\left\{\boldsymbol{\theta}_{\mathrm{M}}\right\} \in \mathbb{R}^{N_{\mathrm{M}}}$. In structural dynamics applications, an uncorrelated zero-mean Gaussian model (i.e. "white noise") is typically selected to represent the prediction error. This model has the advantage of improving tractability of expressions and computations considerably; furthermore, it is supported by the ME principle: it maximizes the entropy with respect to the prediction error $\boldsymbol{\eta}$ (and not with respect to the model parameters $\boldsymbol{\theta}$ ). However, in some applications this white noise model may not be appropriate, for instance when the errors show correlations (e.g. when densely populated sensor grids are used) or a systematic component (e.g. due to modeling error or faulty measurement equipment/setup). Moreover, the magnitude of the errors is often unknown.

In order to avoid incorrect or unsuitable assumptions, and thereby unfairly influencing the Bayesian updating results, one can alternatively make use of the available observed data to try and identify (characteristics of) the prediction error. Several levels of identification are possible; for instance, it can be opted to make a reasonable assumption regarding the model class, and include the error parameters in the Bayesian scheme. Most often, variance parameters of uncorrelated zero-mean Gaussian models are included, see for instance works by Christodoulou and Papadimitriou [35, 143, 144] and Zhang et al. 145], but correlation parameters such as correlation lengths can be included as well [146]. Other similar identification approaches try to estimate (parameters of) a systematic error, see e.g. methods suggested by Kennedy and O'Hagan [54] and Gardoni et al. 147]. 
A second category of identification approaches makes use of Bayesian model class selection (MCS) 130, 131], where the Bayesian inference scheme is applied at model class level to determine, among several alternative model classes, the model class that is most suitable based on the available information. Within this second category of methods, two routes can be followed. Firstly, it could be decided to use MCS to differentiate between several alternative prediction model classes $\mathcal{M}_{\mathrm{M}}$, in an effort to eliminate - or at least reduce - the model (structure) error. This is a very popular approach (see e.g. [148 150]), but still requires the selection of a suitable probabilistic model for the remaining model parameter error. A second alternative approach consists of applying Bayesian model class selection to determine the most suitable probabilistic model class to represent the prediction error based on the information at hand. For further details and applications, the reader is referred to [146, 151].

\subsection{The posterior PDF}

When the prior PDF and likelihood function are determined, equation (21) allows for the updating of the PDFs of the model parameters based on experimental observations of the system. For most practical applications where multiple parameters are involved, computing the joint and marginal PDFs requires solving high-dimensional integrals, therefore use is often made of approximate measures (section 4.5.3) or sampling methods such as Markov chain Monte Carlo (MCMC) methods (section 4.5.4). In selected cases, the posterior PDF can be determined analytically, for instance when conjugate priors are used (as e.g. in section 4.5.2), or numerically, for instance when the number of parameters remains limited to a few.

Once the posterior PDF is computed, estimated or approximated, it can be used to obtain information on the so-called resolution of the parameters through the Bayesian scheme, i.e. how much the uncertainty on the parameters is reduced by the observed data and prior information. A resolution analysis typically includes comparing standard posterior statistics (mean values, standard deviations, covariance matrices), and can be complemented by performing an eigenvalue analysis to help identify well-resolved features or parameter combinations [152].

\subsubsection{Relation to deterministic model updating}

The Bayesian model updating approach also provides important insight into the often ill-posed deterministic model updating problem (section 2.3). This is most easily demonstrated by considering the maximum a posteriori (MAP) estimate, i.e. the peak or mode of the posterior PDF. The MAP estimate is defined as the parameter set that maximizes the posterior PDF, or, equivalently, minimizes the negative log posterior PDF:

$$
\hat{\boldsymbol{\theta}}^{\mathrm{MAP}}=\underset{\boldsymbol{\theta}}{\arg \min } F_{\mathrm{MAP}} \quad \text { where } \quad F_{\mathrm{MAP}}=-\log L(\boldsymbol{\theta} \mid \overline{\mathbf{d}})-\log p(\boldsymbol{\theta})=F_{\mathrm{ML}}+F_{\mathrm{MAPr}}
$$

Assuming a zero-mean Gaussian prediction error characterized by a covariance matrix $\boldsymbol{\Sigma}_{\eta}$, the maximum likelihood (ML) objective function $F_{\mathrm{ML}}$ in the MAP objective function equals:

$$
F_{\mathrm{ML}}=-\log L(\boldsymbol{\theta} \mid \overline{\mathbf{d}})=\frac{1}{2} \boldsymbol{\eta}^{\mathrm{T}} \boldsymbol{\Sigma}_{\eta}^{-1} \boldsymbol{\eta}
$$

It is clear that solving a least squares problem as stated in equation (28) in fact corresponds to solving a classical deterministic model updating problem as defined in equation (5), as the objective function aims to minimize the discrepancy between model predictions and measured data. The weights given to the discrepancies are inversely proportional to the appointed error variances, which corresponds to giving more weight to more accurate data.

Furthermore, it is easy to see that the second term $F_{\mathrm{MAPr}}$ in objective function $F_{\mathrm{MAP}}$ in fact corresponds to a regularization term, based on the prior information available. As such, the deterministic counterpart of the Bayesian inference scheme incorporates regularization in a natural way, without having to revert to re-parameterization or other standard regularization methods that require additional decision-making and may appear heuristic. Moreover, information contained in the prior PDF (e.g. positivity of the parameters) is automatically enforced in the deterministic optimization scheme. 


\subsubsection{Linear model with a Gaussian prior and prediction error}

Suppose the prediction model $\mathbf{G}_{\mathbf{M}}$ is a linear model (in terms of $\boldsymbol{\theta}_{\mathbf{M}}$ ), so that its output can be written as $\mathbf{J} \boldsymbol{\theta}_{\mathrm{M}}$. It is assumed that the probabilistic model of the prediction error $\boldsymbol{\eta}=\overline{\mathbf{d}}-\mathbf{J} \boldsymbol{\theta}_{\mathrm{M}}$ is a zero-mean Gaussian model, characterized by $\boldsymbol{\Sigma}_{\eta}$, which reduces the total parameter set to $\boldsymbol{\theta}=\boldsymbol{\theta}_{\mathrm{M}}$. A priori, the parameter set $\boldsymbol{\theta}$ is assumed to be a Gaussian random variable characterized by a mean value $\boldsymbol{\theta}_{\mathrm{pr}}$ and a prior covariance matrix $\boldsymbol{\Sigma}_{\theta, \mathrm{pr}}$. As both the prior PDF and the likelihood function are Gaussian, the posterior PDF will also have a Gaussian distribution, characterized by a mean value $\boldsymbol{\theta}_{\text {po }}$ and a covariance matrix $\boldsymbol{\Sigma}_{\theta, \text { po }}$, which can easily be verified to be equal to:

$$
\begin{aligned}
\boldsymbol{\theta}_{\mathrm{po}} & =\left(\mathbf{J}^{\mathrm{T}} \boldsymbol{\Sigma}_{\eta}^{-1} \mathbf{J}+\boldsymbol{\Sigma}_{\theta, \mathrm{pr}}^{-1}\right)^{-1}\left(\mathbf{J}^{\mathrm{T}} \boldsymbol{\Sigma}_{\eta}^{-1} \overline{\mathbf{d}}+\boldsymbol{\Sigma}_{\theta, \mathrm{pr}}^{-1} \boldsymbol{\theta}_{\mathrm{pr}}\right) \\
\boldsymbol{\Sigma}_{\theta, \mathrm{po}} & =\left(\mathbf{J}^{\mathrm{T}} \boldsymbol{\Sigma}_{\eta}^{-1} \mathbf{J}+\boldsymbol{\Sigma}_{\theta, \mathrm{pr}}^{-1}\right)^{-1}
\end{aligned}
$$

It is interesting to observe that the posterior mean value is obtained as a weighted average of the data set $\overline{\mathbf{d}}$ and the prior mean value $\boldsymbol{\theta}_{\mathrm{pr}}$, where the relative weights are determined by the prediction model and error covariance, and by the prior covariance, respectively. Moreover, it can be noted that the posterior covariance matrix is in this case independent of the posterior mean value $\boldsymbol{\theta}_{\mathrm{po}}$ and the data $\overline{\mathbf{d}}$.

The expressions for the posterior mean value and covariance matrix in this particular case are equivalent to the Kalman filter equations with optimal Kalman gain [153]. Therefore, this Gaussian linear Bayesian inference scheme can in fact be interpreted as a single-step Kalman filtering procedure. Vice versa, the standard Kalman filter can be seen as a special case of the Bayes filter 154 commonly used in robotics.

\subsubsection{Asymptotic approximations}

Papadimitriou et al. 155] and Beck and Katafygiotis [85] show that, when a large amount of data is available, the posterior PDF can be approximated asymptotically by a Gaussian PDF centered at the maximum a posteriori or MAP point and characterized by covariance matrix $\hat{\boldsymbol{\Sigma}}_{\theta \text {,po }}$, i.e.:

$$
p(\boldsymbol{\theta} \mid \overline{\mathbf{d}}) \approx \mathcal{N}\left(\hat{\boldsymbol{\theta}}^{\mathrm{MAP}}, \hat{\boldsymbol{\Sigma}}_{\theta, \mathrm{po}}\right)
$$

The MAP estimate was defined above in equation (27); the approximate covariance matrix $\hat{\boldsymbol{\Sigma}}_{\theta, \text { po }}$ is computed as the inverse Hessian of the MAP objective function $F_{\mathrm{MAP}}$, evaluated at the MAP point:

$$
\hat{\boldsymbol{\Sigma}}_{\boldsymbol{\theta}, \mathrm{po}}^{-1}=\left.\nabla_{\boldsymbol{\theta}}^{2} F_{\mathrm{MAP}}\right|_{\boldsymbol{\theta}=\hat{\boldsymbol{\theta}}^{\mathrm{MAP}}}
$$

In many optimization algorithms, the Hessian of the objective function $F_{\mathrm{MAP}}$ is computed as a by-product in the solution of the optimization problem defined in equation (27), see section 2.3. As the data set grows, the posterior PDF eventually becomes independent of the prior PDF: the information contained in the prior PDF becomes irrelevant as it ultimately gets overwhelmed by the data. This is a well-known phenomenon in Bayesian inference, described in the Bernstein-von Mises theorem and forming the basis for the original formulation of the asymptotic expressions in 155 .

For a linear prediction model and a Gaussian prior and prediction error model, the asymptotic moments in equations (27) and (32) are exact and identical to the expressions (29) and (30). In other cases, the approximations can be used as a cost-effective alternative to computationally demanding methods (e.g. MCMC sampling), for validation of results obtained by other approaches or as a way to perform an initial reconnaissance of the posterior updating results.

\subsubsection{Sampling methods}

Markov chain Monte Carlo (MCMC) sampling methods [156 158] probably form the most popular sampling techniques in Bayesian inference; most often the Metropolis-Hastings (MH) algorithm [159] is employed to sample the posterior PDF $p(\boldsymbol{\theta} \mid \overline{\mathbf{d}})$. In recent years, a number of methods was developed in order to improve the convergence speed of the classical MH sampling routine. The most commonly implemented 
include adaptive MH MCMC or AM-MCMC 160, delayed rejection or DR-MCMC 161 163, and the hybrid DRAM-MCMC [164]. Another class of improved MCMC approaches involves a gradual or sequential sampling strategy, where the method developed by Au et al. [165] and Beck and Au [166], and the transitional MCMC (or TMCMC) method [167] belong to the most popular. Finally, it should be mentioned that the parallelization of MCMC routines can greatly accelerate the sampling procedure. Interesting methods aimed at Bayesian inference include parallel TMCMC [168], and MCMC approaches based on evolutionary algorithms such as DE-MC [169] and DREAM [170].

\subsection{Example application: $U Q$ in the damage assessment of a reinforced concrete beam}

In this section, Bayesian FE model updating is applied to the reinforced concrete beam example introduced in section 1.3 in order to quantify the uncertainty on the FE model updating results. A prior parameter $\mathrm{PDF}$ is updated to a posterior parameter PDF through the likelihood function, which is constructed based on the observed data $\overline{\mathbf{d}}$ (section 2.4) and the prediction error specifications.

\subsubsection{Prior PDF}

The physical model parameter vector $\boldsymbol{\theta}_{\mathrm{M}}$ corresponds to the set of 10 substructure stiffness parameters of the beam (figure 4). The joint prior PDF is determined using the maximum entropy principle (section 4.3); this leads to a priori independent gamma-distributed variables [142], meaning that the prior PDF is given as:

$$
p\left(\boldsymbol{\theta}_{\mathrm{M}}\right)=\prod_{j=1}^{N_{\mathrm{M}}} p\left(\theta_{\mathrm{M} j}\right)=\prod_{j=1}^{N_{\mathrm{M}}} \frac{\theta_{\mathrm{M} j}^{\alpha_{j}-1}}{\beta_{j}^{\alpha_{j}} \Gamma\left(\alpha_{j}\right)} \exp \left(-\frac{\theta_{\mathrm{M} j}}{\beta_{j}}\right)
$$

Shape factors $\alpha_{j}$ and scale factors $\beta_{j}$ are chosen equal for all parameters; their values are computed based on an assumed prior mean value $\mu=37.5 \mathrm{GPa}$ and standard deviation $\sigma=20 \mathrm{GPa}$.

\subsubsection{Likelihood function and prediction error model}

The likelihood function is constructed using the probabilistic models of the measurement error and the modeling error. In this particular case, some information is available regarding the measurement error $\eta_{\mathrm{D}}$ : the SSI technique applied to extract the modal characteristic from the time-domain vibration data also allows for the estimation of uncertainty bounds on the measurement results. A covariance matrix $\boldsymbol{\Sigma}_{\mathrm{D}}$ characterizing a zero-mean Gaussian measurement error can be estimated from a single set of timedomain data [57]. This measurement error predominantly comprises the estimation error due to the signal processing. For the $\mathrm{RC}$ beam, it is found that these signal processing error variances are relatively small: the maximum coefficient of variation $\sigma / \mu$ reaches about $2 \%$ for the frequencies, and the mode shape variances are 4 to 5 orders of magnitude smaller than the mode shape components. The estimated covariances are not taken into consideration, due to the setup-based data acquisition: the data were constructed by combining experimental data obtained in 6 different setups, where 12 sensors were used in each setup. Therefore, the estimated covariances between mode shape components do not cover the whole range of DOFs along the beam, and prove not very informative.

For the modeling error $\boldsymbol{\eta}_{\mathrm{G}}$, it is known that it is indubitably present, but no information is available regarding its nature or magnitude. It is therefore assumed that the modeling error $\boldsymbol{\eta}_{\mathrm{G}}$ is a fixed zero-mean Gaussian variable characterized by covariance matrix $\boldsymbol{\Sigma}_{\mathrm{G}}$. The modeling errors of all data components are assumed to be statistically independent, so that the $\boldsymbol{\Sigma}_{\mathrm{G}}$ is a diagonal matrix with variances $\sigma_{i}^{2}$ as diagonal elements. The variances are taken proportional to the measured values: for an eigenvalue $\lambda_{r}$, the standard deviation $\sigma_{\lambda, r}$ is assumed to be proportional to the measured value $\bar{\lambda}_{r}$, i.e. $\sigma_{\lambda, r}=\sigma_{\lambda} \bar{\lambda}_{r}$.

For the mode shapes, all $N_{o}$ mode shape components in the vector $\phi_{r}$ are appointed the same standard deviation $\sigma_{\phi, r}$ proportional to the norm of the measured mode shape vector: $\sigma_{\phi, r}=\sigma_{\phi}\left\|\bar{\phi}_{r}\right\|$. This is done to avoid appointing very small standard deviations (i.e. large weights) to mode shape components close to zero. Moreover, this assumption automatically compensates for the number of mode shape components. The standard deviation can be rewritten as $\sigma_{\phi, r}=\sigma_{\phi} \sqrt{N_{o}} \bar{\phi}_{r}^{\mathrm{RMS}}$ with $\bar{\phi}_{r}^{\mathrm{RMS}}$ the root mean square (RMS) 
or quadratic mean value of the mode shape vector which can be interpreted as a measure of the magnitude of the mode shape. As more components are added for the same mode, the standard deviation increases (and weight given to the components therefore decreases), which avoids that the mode shape content would eventually dominate the frequency content as more mode components are added to the data set. Many authors explicitly compensate for this effect (e.g. by multiplying standard deviations by $N_{o}$ ).

In the current application, the dimensionless parameters $\sigma_{\lambda}$ and $\sigma_{\phi}$ are both taken equal to 0.01 . In other words, the modeling error is assumed to cause up to one percent deviation on the nominal model prediction values; this can be interpreted as an adopted model confidence measure.

Using these assumptions, the likelihood function is constructed as:

$$
L\left(\boldsymbol{\theta}_{\mathrm{M}} \mid \overline{\mathbf{d}}\right) \propto\left(\operatorname{det}\left(\boldsymbol{\Sigma}_{\mathrm{D}}+\boldsymbol{\Sigma}_{\mathrm{G}}\right)\right)^{-1 / 2} \exp \left[-\frac{1}{2} F_{\mathrm{ML}}\right]
$$

where the maximum likelihood function $F_{\mathrm{ML}}$ is equal to:

$$
\begin{aligned}
F_{\mathrm{ML}} & =\left(\boldsymbol{\eta}_{\mathrm{D}}+\boldsymbol{\eta}_{\mathrm{G}}\right)^{\mathrm{T}}\left(\boldsymbol{\Sigma}_{\mathrm{D}}+\boldsymbol{\Sigma}_{\mathrm{G}}\right)^{-1}\left(\boldsymbol{\eta}_{\mathrm{D}}+\boldsymbol{\eta}_{\mathrm{G}}\right) \\
& =\left(\mathbf{G}\left(\boldsymbol{\theta}_{\mathrm{M}}\right)-\overline{\mathbf{d}}\right)^{\mathrm{T}}\left(\boldsymbol{\Sigma}_{\mathrm{D}}+\boldsymbol{\Sigma}_{\mathrm{G}}\right)^{-1}\left(\mathbf{G}\left(\boldsymbol{\theta}_{\mathrm{M}}\right)-\overline{\mathbf{d}}\right)
\end{aligned}
$$

\subsubsection{Results of the Bayesian updating scheme}

The joint posterior PDF is sampled using a Markov chain Monte Carlo method, where the MetropolisHastings algorithm is implemented (section 4.5.4). Convergence is reached after about 300000 samples; these are used to estimate the posterior marginal densities of all model parameters using kernel density estimation.

In figure6a, a contour plot of the resulting normalized marginal posterior PDFs of the 10 model parameters (multiplied with the moment of inertia $I$ to correspond to the bending stiffness $E I$ ) is shown; figure $6 \mathrm{~b}$ shows the PDFs of the second and eighth substructure stiffnesses. The Bayesian scheme clearly succeeds in identifying and localizing the damage at about $4 \mathrm{~m}$; the extent of the maximum a posteriori stiffness reduction corresponds quite well to the deterministic results. The stiffness parameters towards the outer ends of the beam are quite poorly resolved for both damage states, which means in these areas very little information is gained from the experimental data. A slightly higher resolution is found in the damaged areas; this can be explained by the fact that the damage induces higher modal curvatures in these zones. Therefore, the experimental data are more sensitive to stiffness changes in these areas and provide more information for the model calibration scheme.

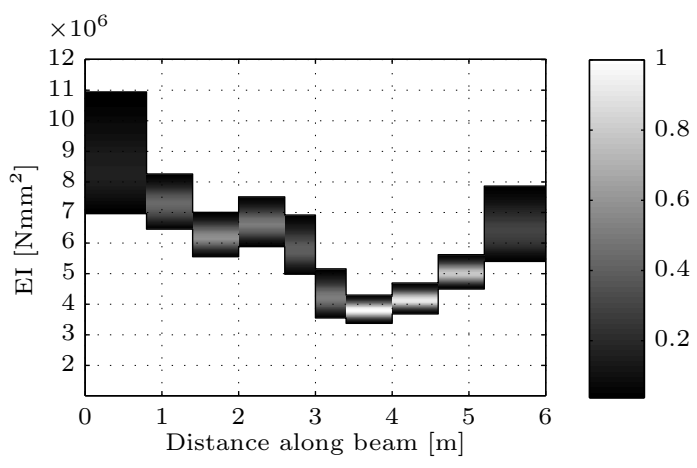

(a)

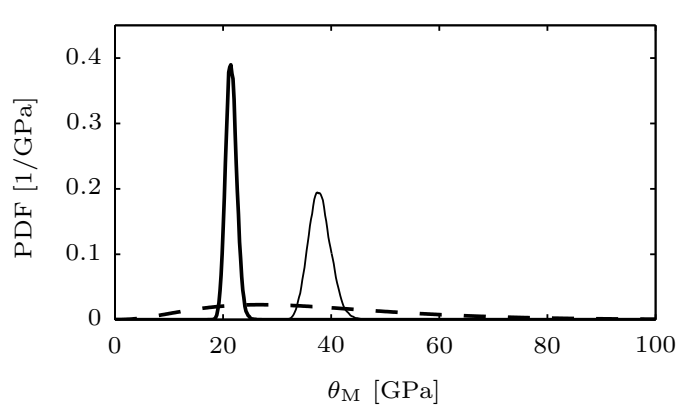

(b)

Figure 6: (a) Contour plots of the normalized marginal posterior PDFs, within their 99\% confidence intervals, of the bending stiffness along the beam, and (b) prior (dashed line) and posterior PDF of substructure stiffnesses $\theta_{\mathrm{M} 2}$ (thin solid line) and $\theta_{\mathrm{M} 8}($ thick solid line). 


\section{Fuzzy FE model updating}

Even though the probabilistic approach is generally seen as the most comprehensive technique to effectively deal with uncertainties, it is often stated that it is not particularly appropriate for modeling epistemic uncertainty [171, 172]. The argumentation behind this relates to the specification of the (joint) PDFs describing the uncertain quantities: it is argued that sufficient qualitative information for composing a truthful and representative probabilistic model is seldom available. For instance, parameter values are often uncertain in the sense that they are known to be "about" a certain value or within a certain interval, and often even less is known regarding possible interactions or dependencies between quantities. Several methods such as bootstrap or jackknife resampling [173] and the principle of maximum entropy (section 4.3) have been suggested as effective means to deal with insufficient information, but it is often argued that in many cases the selection of PDFs remains quite arbitrary. This reasoning has stimulated the development of a wide array of non-probabilistic (and mixed) uncertainty modeling approaches.

The literature regarding alternative strategies for modeling (epistemic) uncertainty is well-established, see for instance reviews by Helton et al. 174], Oberguggenberger [175], Möller and Beer 171], Ross et al. [176] and Bernardini and Tonon [177], comparative studies and discussions by Bae et al. [178], Ferson [179], Oberkampf [180], Elishakoff [181 183] and Langley [184], and a special edition of the Journal for Applied Mathematics and Mechanics (Zeitschrift für Angewandte Mathematik und Mechanik) [185].

Most non-probabilistic methods are based on interval analysis, where uncertainty on variables is represented by a certain value range, which is then propagated to (interval-valued) outputs of interest. One of the most popular extensions of interval analysis is fuzzy set theory, originally attributed to Zadeh [186]. It provides a method for uncertainty modeling in situations where besides interval bounds, confidence values or degrees of belief regarding the uncertain quantities are available (or desired). In fuzzy set theory, the classical binary concept of a set (i.e. an element either belongs to a set or not) is replaced by a more intuitive description of sets, where membership is gradual and determined by a so-called membership function $\mu_{\widetilde{x}}$ (figure 7a). The power of the fuzzy method lies in the gradual description of membership, which can be interpreted differently depending on the specific application. One of the most common interpretations of membership is that of a measure of uncertainty or plausibility, since this provides a framework to apply fuzzy set theory in the interest of uncertainty quantification (UQ). Therefore, in the present work, only this interpretation of membership will be considered. For further discussions on fuzzy semantics the reader is referred to the works by Dubois and Prade [187], Degrauwe [188] and Oberguggenberger [189].

Reference works on fuzzy set theory were developed by Kaufmann [190], Zadeh [191] and Zimmerman [192]. In a structural engineering context, UQ in structural (FE) analysis and structural design form the most prominent application areas of fuzzy set theory; reviews can be retrieved in Degrauwe [188] and Beer [193, 194], respectively. Convex models of uncertainty were considered by Ben-Haim et al. [16] in a method developed for evaluating the usability of models based on the robustness to uncertainties of decisions resulting from the model predictions. In the same line of thought, a method was developed by Ben-Haim [195] that allows assessing the value of information provided by model updating. Applications of fuzzy set theory in structural analysis can be found e.g. in [196 198], applications in structural design are retrieved in [199201]. Fuzzy set theory was furthermore applied extensively for UQ in structural dynamics, e.g. by Moens et al. 202], Degrauwe et al. 203], Adhikari et al. 204], Chowdhury and Adhikari [205] and Khodaparast et al. 206]. Another popular application area is formed by fuzzy reliability analysis 207 211].

Only in recent years, fuzzy set theory was introduced for UQ in inverse modeling; for instance, Haag and Hanss et al. 212 214 developed a method to estimate fuzzy model parameters based on fuzzy observations, using predominantly forward calculations. In the present work, two different approaches are discussed: the classical nested approach, where fuzzy set theory is applied directly to the inverse problem, and a direct approach that only requires forward computations. The following section 5.1 forms a preamble to this, briefly presenting the specifics of fuzzy set theory and fuzzy computations.

\subsection{Fuzzy sets and fuzzy numbers}

The fuzzy approach consists in modeling every uncertain quantity $x$ as a fuzzy number $\widetilde{x}$, characterized by a (convex) membership function $\mu_{\widetilde{x}}$ (figure $7 \mathrm{a}$ ), and propagating these fuzzy inputs to the output quantities 
of interest. This is usually done through discretization of the fuzzy numbers into a set of $N_{\alpha}$ intervals or so-called $\alpha$-cuts $[\widetilde{x}]_{\alpha}$ (figure $\left.7 \mathrm{~b}\right)$; as such, a fuzzy number in fact represents a set of nested intervals, each reflecting a different plausibility level $\alpha$. Typically, fuzzy numbers are normalized so that all membership values $\alpha$ lie within the interval $[0,1]$.

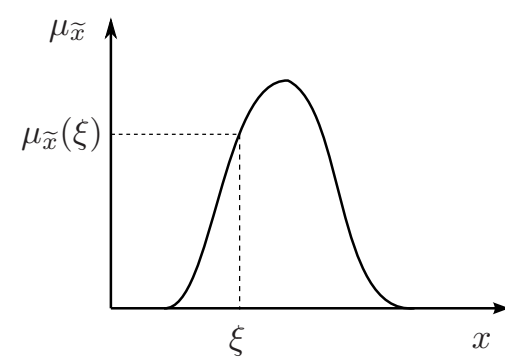

(a)

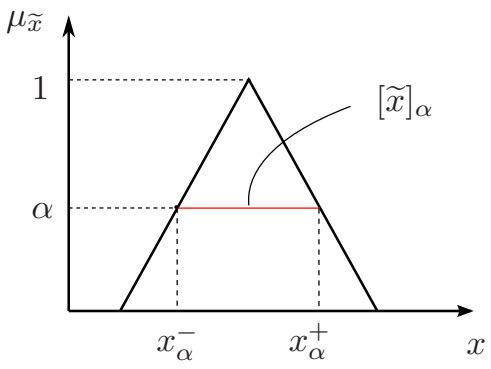

(b)

Figure 7: (a) An example of a membership function $\mu_{\widetilde{x}}$ and (b) an $\alpha$-cut of a triangular fuzzy number $\widetilde{x}$ at level $\alpha$.

Solving a fuzzy problem now consists in solving the following set of interval expressions:

$$
\forall \alpha \in[0,1]: \quad[\widetilde{y}]_{\alpha}=f\left(\left[\widetilde{x}_{1}\right]_{\alpha}, \ldots,\left[\widetilde{x}_{N}\right]_{\alpha}\right)
$$

where an interval expression is defined as:

$$
[\widetilde{y}]=f\left(\left[x_{1}\right], \ldots,\left[x_{N}\right]\right)=\left\{y=f\left(x_{1}, \ldots, x_{N}\right) \mid x_{i} \in\left[x_{i}\right], i=1, \ldots, N\right\}
$$

The challenge associated with discretized fuzzy problems (or any interval-based problem in general) lies primarily in solving the interval problem defined in equation (37), as the two most important solution methods both deal with substantial limitations. The first approach makes use of interval arithmetic, which acts directly on the mathematical operators by generalizing them to interval operators. Although this approach is computationally efficient, it exhibits the well-known dependency problem, where variables that occur multiple times are interpreted as independent from one another, resulting in gross overestimations in the obtained uncertainty bounds 215]. Other disadvantages of interval arithmetic arise in the actual implementation of the method: generalizing implicitly defined operators (e.g. solving a differential equation or an optimization problem) to interval operators often poses major challenges, and furthermore prevents the use of existing software packages.

The second approach for solving interval problems (with continuous objective functions) aims to determine upper and lower bounds of the output quantities by reformulating the interval problem in equation (37) as a constrained optimization problem:

$$
\forall \alpha \in[0,1]: \quad\left\{\begin{array}{l}
y_{\alpha}^{-}=\min f\left(x_{1}, \ldots, x_{N}\right) \\
y_{\alpha}^{+}=\max f\left(x_{1}, \ldots, x_{N}\right)
\end{array} \quad \text { subject to } x_{i} \in\left[\widetilde{x}_{i}\right]_{\alpha}\right.
$$

or, in vector notation:

$$
\forall \alpha \in[0,1]: \quad\left\{\begin{array}{l}
y_{\alpha}^{-}=\min f(\mathbf{x}) \\
y_{\alpha}^{+}=\max f(\mathbf{x})
\end{array} \quad \text { subject to } \mathbf{x} \in[\widetilde{\mathbf{x}}]_{\alpha}\right.
$$

Through this reformulation, all function evaluations become deterministic, meaning that all types of functions can be tackled, and an exact solution is obtained. Another advantage of the method is that - contrary to the interval arithmetic approach - it is a non-intrusive method, meaning that all existing software for evaluating the objective function can be employed without adaptations. An important disadvantage of this method lies in the associated computational cost: optimization algorithms usually require a large number 
of function evaluations.

The optimization approach is most often adopted as solution strategy for expression (37), as the advantages of this approach usually far outweigh the benefits of using interval arithmetic. Several methods can be applied to solve the constrained optimization problem (40), where general purpose global or local optimization algorithms [216, 217] and vertex methods belong to the most popular. Vertex methods 218] such as the (general and reduced) transformation method [219, 220] aim to find the optima of equation (40) based on evaluating vertices of the hypercube defined through the $\alpha$-cut intervals. These methods require a monotonic objective function in order to obtain correct results, in which case very efficient algorithms (e.g. the short transformation method [221]) may be obtained.

In recent years, several improved vertex methods were developed to tackle nonmonotonic function behavior in fuzzy problems, such as the maximum possibility search method by Choi et al. [199], the Taylor's expansion with extrema management method by Massa et al. [201], the gradual number method by Untiedt and Lodwick [222] and the gradual $\alpha$-level decreasing $(\mathrm{G} \alpha \mathrm{D})$ algorithm by Degrauwe et al. [223]. A substantial limitation of practically all vertex methods (except for the short transformation method) is that they are only applicable to problems with a relatively limited number of uncertain variables $N$, say $N<20$, as they usually require a minimum of $2^{N}$ function evaluations.

In cases when problem-specific vertex methods cannot be applied (e.g. for a large amount of input variables), and general optimization algorithms become too costly (e.g. when a large number of expensive function evaluations are required), response surface methods [224] provide a means to obtain a cost-efficient approximate solution of the fuzzy interval problem. This is done by replacing the expensive function operator by an approximate response surface, which is usually constructed through interpolation (e.g. Kriging 225]). The reader is referred to 206, 226 230] for details on the response surface methods for fuzzy problems and further applications.

\subsection{Application of fuzzy set theory for model updating}

In this section, two different approaches are presented to employ fuzzy set theory for uncertainty quantification purposes in model updating. The main difference between the two strategies lies in the interpretation of the objective of the uncertainty quantification problem, which can consist in either (1) finding the uncertainty range on the deterministic model updating results, given the uncertainty on the data and model predictions, or (2) assessing the uncertainty on the model parameters, given the uncertainty on the data and model predictions. To both these goals, a suitable fuzzy solution strategy is presented in this section [151].

In either case, the first and most determining step consists in translating the existing uncertainties into fuzzy input variables in such a way that the available information is modeled in a representative and comprehensive fashion. As mentioned previously in section 3.3. the uncertainties or errors occurring in model updating can be categorized into the modeling error $\boldsymbol{\eta}_{\mathrm{G}}$ and the measurement error $\boldsymbol{\eta}_{\mathrm{D}}$. Therefore, uncertainty regarding the model predictions and measurements can be modeled by modeling the respective errors, or the total prediction error, as fuzzy numbers:

$$
\widetilde{\boldsymbol{\eta}}=\widetilde{\boldsymbol{\eta}}_{\mathrm{G}}+\widetilde{\boldsymbol{\eta}}_{\mathrm{D}}
$$

In order to avoid modeling both errors as fuzzy numbers - which could be cumbersome as e.g. the modeling error is dependent on the considered parameter set - it could be suggested to shift all uncertainty to the data. As such only the experimental data set needs to be modeled as a fuzzy number:

$$
\widetilde{\boldsymbol{\eta}}=\widetilde{\boldsymbol{\eta}}_{\mathrm{G}}+\widetilde{\boldsymbol{\eta}}_{\mathrm{D}}=\mathbf{G}_{\mathrm{M}}\left(\boldsymbol{\theta}_{\mathrm{M}}\right)-\widetilde{\overline{\mathbf{d}}}
$$

In the interest of clarity, a fuzzy experimental data set will be denoted as $\widetilde{\mathbf{d}}$ in the following.

Although some generic method have been proposed (e.g. 231, 232]), in practice a wide variety of approaches is used to specify a suitable membership function. In some cases, a membership function is constructed based on some probabilistic measures such as estimated means and standard deviations or confidence intervals, or even based on a complete (estimated) probability density function. It should in any case be clear that the resulting membership values cannot be interpreted as probabilities. In most practical 
applications, however, the membership function is appointed some functional (linear) form, based on the available information, leading e.g. to triangular or trapezoidal fuzzy numbers.

\subsubsection{Nested fuzzy model updating}

Suppose the aim is to employ fuzzy set theory as presented above to quantify the effect of uncertainties on the outcome of the deterministic updating process as defined in equation (77). For notational efficiency, this updating problem will be denoted as $\boldsymbol{\theta}_{\mathrm{M}}^{*}=g_{\mathrm{M}}(\boldsymbol{\eta})$, or, assuming a fixed model class, $\boldsymbol{\theta}_{\mathrm{M}}^{*}=g_{\mathrm{M}}(\overline{\mathbf{d}})$. Introducing this expression directly into equation (37) yields:

$$
\forall \alpha \in[0,1]: \quad\left[\widetilde{\boldsymbol{\theta}}_{\mathrm{M}}^{*}\right]_{\alpha}=g_{\mathrm{M}}\left(\left[\widetilde{d}_{1}\right]_{\alpha}, \ldots,\left[\widetilde{d}_{N}\right]_{\alpha}\right)
$$

As this problem entails a multidimensional output vector $\boldsymbol{\theta}_{\mathrm{M}}^{*} \in \mathbb{R}^{N_{\mathrm{M}}}$, it can be reformulated as the following optimization problem:

$$
\forall j, k:\left\{\begin{array}{l}
\left(\widetilde{\theta}_{\mathrm{M} k}^{*}\right)_{\alpha_{j}}^{-}=\min _{\overline{\mathbf{d}}}\left[g_{\mathrm{M}}(\overline{\mathbf{d}})\right]_{k} \\
\left(\widetilde{\theta}_{\mathrm{M} k}^{*}\right)_{\alpha_{j}}^{+}=\max _{\overline{\mathbf{d}}}\left[g_{\mathrm{M}}(\overline{\mathbf{d}})\right]_{k}
\end{array} \quad \text { subject to } \overline{\mathbf{d}} \in[\widetilde{\mathbf{d}}]_{\alpha_{j}}\right.
$$

where $\overline{\mathbf{d}} \in \mathbb{R}^{N}, j=1, \ldots, N_{\alpha}$ and $k=1, \ldots, N_{\mathrm{M}}$. This means $2 N_{\mathrm{M}}$ regular optimizations are required at each $\alpha$-level, except at the top level where a single optimization suffices; the total number of required optimization routines therefore equals $N_{\mathrm{M}}\left(2 N_{\alpha}-1\right)$. As each function evaluation $g_{\mathrm{M}}(\overline{\mathbf{d}})$ also involves an optimization, equation (44) in fact corresponds to a nested optimization scheme, hence the name of this approach. The inner optimization determines the optimal model parameters for a certain set of experimental data; the outer optimization problem determines the optimal sets of experimental data, within the defined $\alpha$-level intervals, that result in the extreme values of the updated model parameters.

It should be noted that solving the outer fuzzy model updating problem is non-trivial. Due to the high number of input variables (here: the number of observations $N$ ), most vertex methods are not applicable as they require evaluating the objective function for $2^{N}$ binary combinations of the lower and upper interval bounds of the $N$ fuzzy input variables. Application of the short transformation method would not yield accurate results as the objective function $g_{\mathrm{M}}$ is in general not a monotonic function, but exhibits nonsmooth behavior with many local optima. This means that in order to obtain an accurate solution, only general-purpose optimization routines remain; however, in this case the required computational effort is substantial. The computation time can be reduced drastically by taking advantage of the fact that the fuzzy problem can be parallelized very easily, as all $N_{\mathrm{M}}\left(2 N_{\alpha}-1\right)$ outer optimization routines are completely independent from one another; the fuzzy problem is therefore said to be embarrassingly parallel. Additionally, computational effort could be lessened further by exploiting the fact that most likely, many of the required forward computations involve solving identical forward problems.

\subsubsection{Direct fuzzy model updating}

When the aim of the UQ problem is instead to determine the uncertainty on the model parameters, given the uncertainty on the model predictions and experimental data, it is easy to see that the inverse problem defined in equation (77) in fact does not need to be considered. The problem can be solved by searching, at each $\alpha$-level, the extremal model parameters that result in a model output $\mathbf{y}=\mathbf{G}_{\mathrm{M}}\left(\boldsymbol{\theta}_{\mathrm{M}}\right)$ within the bounds determined by the $\alpha$-cut interval $[\widetilde{\mathbf{d}}]_{\alpha_{j}}$. This yields the following optimization problem:

$$
\forall j, k:\left\{\begin{array}{l}
\left(\widetilde{\theta}_{\mathrm{M} k}^{*}\right)_{\alpha_{j}}^{-}=\min _{\boldsymbol{\theta}_{\mathrm{M}}} \theta_{\mathrm{M} k} \\
\left(\widetilde{\theta}_{\mathrm{M} k}^{*}\right)_{\alpha_{j}}^{+}=\max _{\boldsymbol{\theta}_{\mathrm{M}}} \theta_{\mathrm{M} k}
\end{array} \quad \text { subject to } \mathbf{G}_{\mathrm{M}}\left(\boldsymbol{\theta}_{\mathrm{M}}\right) \in[\widetilde{\mathbf{d}}]_{\alpha_{j}}\right.
$$

where $\mathbf{G}_{\mathrm{M}}\left(\boldsymbol{\theta}_{\mathrm{M}}\right) \in \mathbb{R}^{N}, j=1, \ldots, N_{\alpha}$ and $k=1, \ldots, N_{\mathrm{M}}$. This expression clearly makes use of forward computations only. Intuitively, it would be easy to presume that this strategy provides a cost-efficient alternative to the previously elaborated nested approach, but the significant conceptual difference between 
both methods should be noted. The direct formulation in equation (45) only considers physically feasible points in the parameter space that satisfy the prediction model exactly, meaning that a one-to-one mapping from parameter space to data (or error) space is inherently pertained. In other words, the residual between the measured and computed data is always exactly zero. In the nested approach, however, the mapping $g_{\mathrm{M}}$ from data set to model parameters is not exact; rather, the residual between the two quantities is minimized. As such, both strategies solve fundamentally different problems; the most suitable method is dependent on the objective of the uncertainty quantification procedure.

\subsubsection{Dependency considerations}

A substantial issue associated with standard fuzzy set theory is its intrinsic incapability to take dependency or interaction between fuzzy input variables into account. This means that fuzzy modeling always yields the maximal (worst case) range on the output variables at each $\alpha$-level, as it is implicitly assumed that any combination of input variable values is equally plausible. In other words, solving e.g. the problem in (44) produces the extreme values of the objective function, regardless of the (known) feasibility of the input variable set. In FE model updating, for instance, an optimum may be found for a data set that includes very large differences between neighboring mode shape displacements, which is physically highly unlikely.

One possible approach to overcome this problem is to make use of so-called interactive fuzzy numbers [188, 233, 234], where dependency between variables is taken into account by modifying the joint membership function to exclude physically infeasible or unlikely input combinations at each $\alpha$-level. This in fact corresponds to altering the $N$-dimensional box bounds in optimization problem (44) (or, more generally, in problem (40)) to general convex bounds.

An additional dependency issue that is often overlooked when applying fuzzy modeling to multiple-output problems, such as model updating problems, is that possible dependencies or interactions between output variables are also neglected: the result of the fuzzy calculation is a number of independent fuzzy outputs. Take, for instance, the optimization problem (44) for model updating problems. It is clear that each model parameter is optimized individually, while all other parameters can vary unrestrictedly, which may result in non-physically feasible parameter combinations. This issue can be circumvented by enforcing additional constraints on the updating parameters.

\subsection{Example application: UQ in the damage assessment of a reinforced concrete beam}

In this section, the application introduced in section 1.3 is resumed in order to illustrate how fuzzy set theory can be used to assess the effect of uncertainties on the results of a FE model updating scheme. In order to take uncertainty into account, the experimental data $\overline{\mathbf{d}}$ are modeled as symmetric fuzzy numbers $\widetilde{\mathbf{d}}$ with triangular membership functions, where the nominal values (i.e. with a membership value equal to one) are taken to be the measured data $\overline{\mathbf{d}}$. The support intervals (at $\alpha=0$ ) are chosen based on the known measurement error variance and an assumed value for the modeling error. The interval bounds at the other $\alpha$-levels are interpolated linearly, leading to the following $\alpha$-cut expression for an observation $\widetilde{d}_{i}$ :

$$
\left[\widetilde{d}_{i}\right]_{\alpha}=\left[\bar{d}_{i}-\Delta_{\alpha, i}^{\mathrm{D}}-\Delta_{\alpha, i}^{\mathrm{G}}, \bar{d}_{i}+\Delta_{\alpha, i}^{\mathrm{D}}+\Delta_{\alpha, i}^{\mathrm{G}}\right]
$$

The respective contributions of the measurement error and modeling error, $\Delta_{\alpha, i}^{\mathrm{D}}$ and $\Delta_{\alpha, i}^{\mathrm{G}}$, are defined as:

$$
\begin{aligned}
& \Delta_{\alpha, i}^{\mathrm{D}}=(1-\alpha) z \bar{\sigma}_{i} \\
& \left\{\begin{array}{cl}
\Delta_{\alpha, i}^{\mathrm{G}}=(1-\alpha) z \sigma_{i} \bar{\lambda}_{i} & \text { for } \quad \bar{d}_{i}=\bar{\lambda}_{i} \\
\Delta_{\alpha, i}^{\mathrm{G}}=(1-\alpha) z \sigma_{i}\left\|\bar{\phi}_{r}\right\| & \text { for } \quad \bar{d}_{i}=\bar{\phi}_{r, k}
\end{array}\right.
\end{aligned}
$$

In these expressions, $\bar{\sigma}_{i}$ represents the estimated standard deviation of the measurement error associated with $\bar{d}_{i} ; \sigma_{i}$ is a parameter that determines the magnitude of the modeling error, here all $\sigma_{i}$ are taken equal to 0.01 . $z=\Phi^{-1}(1-\beta / 2)=2.58$ is a factor to incorporate the $99 \%$ confidence interval of the errors into the $\alpha$-cut interval; $\Phi^{-1}$ denotes the normal inverse CDF, and $\beta=0.01$.

In order to illustrate the above discourse regarding the two alternative strategies to fuzzy model updating, both the nested and the direct formulation are applied for UQ in the particular case of the RC beam. 


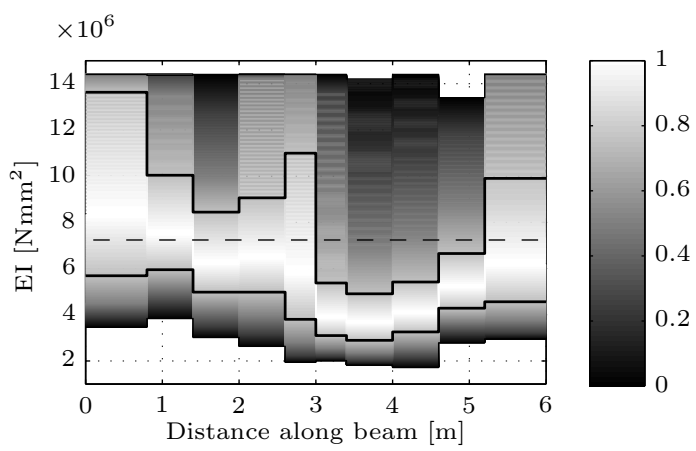

(a)

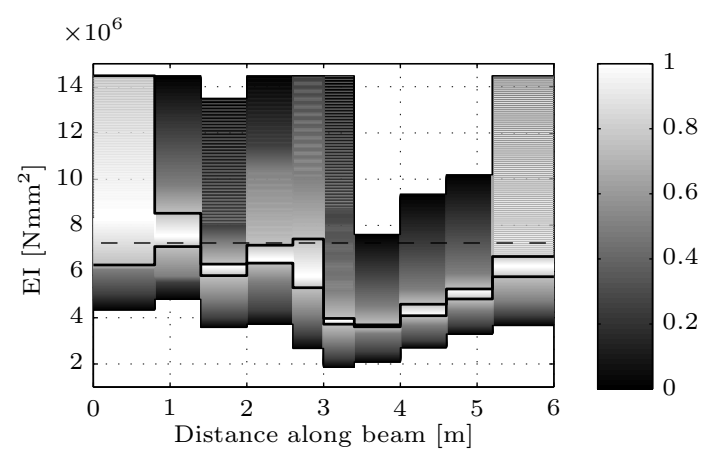

(b)

Figure 8: Contour plots of the membership functions of the resulting bending stiffness parameters for (a) the nested approach and (b) the direct approach. The black solid line represents $\alpha$-level $\alpha=0.75$.

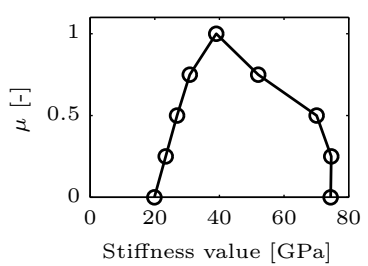

(a) $\widetilde{\theta}_{\text {M2 }}^{\text {nested }}$

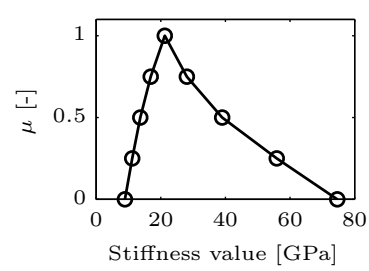

(b) $\widetilde{\theta}_{\mathrm{M} 8}^{\text {nested }}$

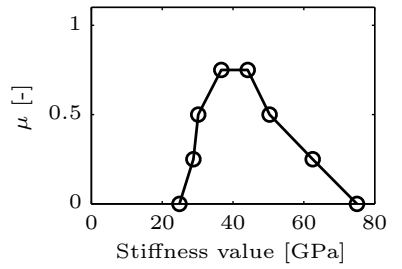

(c) $\widetilde{\theta}_{\mathrm{M} 2}^{\text {direct }}$

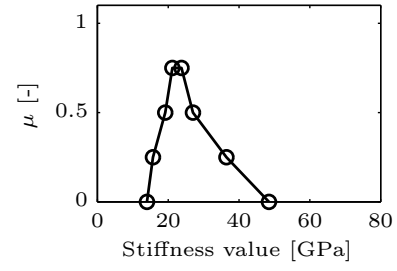

(d) $\widetilde{\theta}_{\mathrm{M} 8}^{\text {direct }}$

Figure 9: Membership functions of the second and eighth stiffness value, both for the nested and the direct approach.

\subsubsection{Nested formulation}

In view of the comments above, some changes are made to the original deterministic FE model updating routine as described in section 2.4. Firstly, lower and upper constraints are imposed on the values of the model stiffness parameters, in this case $\theta_{\mathrm{M} k} \in\left[0,2 \theta_{\mathrm{M} 0}\right]$, where $\theta_{\mathrm{M} 0}=37.5 \mathrm{GPa}$. As such, it is avoided that a single stiffness parameter for instance tends to a very high and unrealistic value due to the fact that it is maximized individually without simultaneously considering the other stiffness values. Theoretically, a very high stiffness value in a certain area of the structure could be compensated by unrealistically low stiffness values in other areas, and vice versa.

Instead of the stiffness values themselves, correction factors are considered in order to improve the stability of the deterministic updating routine. A correction factor $a_{\mathrm{M} k}$ is defined from $\theta_{\mathrm{M} k}=\theta_{\mathrm{M} 0}\left(1-a_{\mathrm{M} k}\right)$. The lower and upper constraints for the correction factors are now equal to -1 and 1 , respectively. The scaling to dimensionless parameters removes any effect of possible different orders of magnitude between the individual updating variables, and ensures physically meaningful system matrices.

In the fuzzy FE model updating scheme, $N_{\alpha}=5 \alpha$-levels are considered, resulting in a total number of 90 outer optimization routines, each requiring a number of calls to the objective function $g_{\mathrm{M}}$, each of which in its turn requires a number of forward computations of the FE model. For the outer optimization routines, the active set algorithm is selected. In order to solve expression (44) as expeditiously as possible, the parallelization possibilities are maximally exploited. It is found that about 160000 deterministic updating schemes are needed, each of which in turn requires on average 15 forward FE computations, bringing the total amount of required forward computations to about $2.5 \times 10^{6}$.

In figure 8a contour plots of the resulting membership functions of the stiffness parameters (multiplied by the moment of inertia) are shown; figures 9 and 9 b show membership functions of the second and eighth stiffness parameter (figure 4). It is immediately clear that at the lower $\alpha$-levels, the upper constraints of the stiffness parameters are reached for almost all parameters, which clearly shows the necessity of the bound constraints on the stiffness values. Overall, a very similar stiffness pattern is found as in the deterministic 
case (figure 5). Further, larger uncertainties are found on the outer ends and in the center of the beam, whereas smaller uncertainties are observed in the damaged zone around $4 \mathrm{~m}$ (figure 8a $)$. These findings are consistent with the expectations and the previously obtained results.

\subsubsection{Direct formulation}

In expression (45), the constraints now depend on the values of the model parameters. It is important to note that for the mode shape components a suitable scaling needs to be carried out: the computed mode shapes are scaled so that a least squares fit is obtained with the corresponding upper or lower bound of $[\widetilde{\mathbf{d}}]_{\alpha_{j}}$. Since triangular membership functions are adopted for the uncertain data $\mathbf{d}$, the computations are performed for the lowest four $\alpha$-levels; for the top $\alpha$-level no physically feasible solutions are found. This means in total 80 parallel optimization routines are required, where the SQP algorithm with standard tolerances is applied [45]. In total, the optimization only requires about 80000 forward calculations of the FE model, considerably less than the nested implementation.

Examining the results of the direct approach shown in figures $8 \mathrm{~b}$, 9r and $9 \mathrm{~d}$, it can be stated that the trends found using the direct strategy are very similar to those obtained by means of the nested approach: larger uncertainty at the outer ends of the beam and reduced uncertainty in damaged areas. However, clearly less wide bounds are obtained compared to the nested implementation, due to fact that only feasible points are considered in this approach, as discussed above. This difference in uncertainty bounds is found to increase as the data intervals get narrower, that is, at higher $\alpha$-levels.

\section{Conclusions}

This work addresses the treatment of uncertainties in model updating problems, with a specific focus on vibration-based FE model updating in structural mechanics. The basic objective of model updating is to calibrate parameters of numerical models based on experimentally observed data; in FE model updating typically use is made of modal characteristics such as natural frequencies and mode shapes to update stiffness parameters along a structure. As such, FE model updating allows for the detection, localization and quantification of existing structural damage, and is therefore a popular methodology in a SHM context. The inverse model updating problem is usually described as an optimization problem, where the goal is to determine the set of model parameters that results in an optimal fit between the numerical model output and the observational data. A detailed investigation of this optimization problem in section 2 revealed its proneness to ill-posedness and ill-conditioning, indicating the sensitivity of the deterministic model updating problem to ever-present measurement uncertainty and modeling uncertainty, as discussed in section 3 .

Out of the multitude of available uncertainty quantification approaches, two of the most popular methods are treated in detail: a probabilistic method based on Bayesian inference (section 4), and a non-probabilistic interval-based method based on fuzzy set theory (section 5). Both approaches are fundamentally different in nature, due to their contrasting interpretations of uncertainty; this implies their results cannot be compared quantitatively, only qualitatively. However, both methods exhibit distinct merits and drawbacks concerning their application for UQ in model updating. The fuzzy approach is in general not very complex to implement and the results are often much more straightforward and intuitive to interpret than for the Bayesian method. When the results obtained by this latter approach are properly processed, however, they provide detailed information regarding the resulting uncertainty of the quantities of interest. Actually obtaining results of the probabilistic method can be quite challenging and computationally demanding, e.g. when methods such as MCMC sampling are to be employed. The fuzzy approach, on the other hand, only yields a worst-case scenario as dependencies between model parameters and/or experimental data cannot be accounted for, contrary to the Bayesian approach, where interactions are automatically included. The computational efficiency of both approaches is dependent on the adopted solution method and the exploitation of parallelization possibilities.

In general, it can be concluded that the most suitable method is dependent on the nature and size of the model updating problem, the available information, and - most importantly - the desired end purpose of the uncertainty quantification procedure. When the objective is to study the worst-case effect of uncertainty 
on model updating results, e.g. for design or sizing purposes, the fuzzy approach seems a suitable means to this end. In other cases, when detailed information is required regarding the resolution and interaction of the parameters, the Bayesian approach is most suited.

\section{Acknowledgments}

The research presented in this paper has been performed within the framework of the project G.0738.11 "Inverse identification of wind loads on structures", funded by the Research Foundation Flanders (FWO), Belgium. Their financial support is gratefully acknowledged.

The authors are members of the KU Leuven - BOF PFV/10/002 OPTEC - Optimization in Engineering Center.

\section{References}

[1] J. Mottershead, M. Friswell, Model updating in structural dynamics: a survey, Journal of Sound and Vibration 167(2) (1993) 347-375.

[2] M. Friswell, J. Mottershead, Finite element model updating in structural dynamics, Kluwer Academic Publishers, Dordrecht, The Netherlands, 1995.

[3] C.-P. Fritzen, D. Jennewein, T. Kiefer, Damage detection based on model updating methods, Mechanical Systems and Signal Processing 12 (1) (1998) 163-186.

[4] M. Imregun, W. J. Visser, A review of model updating techniques, The Shock and Vibration Digest 23 (1) (1991) 9-20.

[5] A. Teughels, G. De Roeck, Damage detection and parameter identification by finite element model updating Archives of Computational Methods in Engineering 12 (2) (2005) 123-164. URL http://www . cimne.com/tiendaCIMNE/productoscon.asp?id_prod=1151

[6] C. Boller, F. Chang, Y. Fujino (Eds.), Encyclopedia of Structural Health Monitoring, John Wiley \& Sons, 2009.

[7] J. Brownjohn, Structural health monitoring of civil infrastructure, Philosophical Transactions of the Royal Society A Mathematical, Physical and Engineering Sciences 365 (1851) (2007) 589-622.

[8] H. Sohn, C. Farrar, F. Hemez, D. Shunk, D. Stinemates, B. Nadler, A review of structural health monitoring literature: 1996-2001, Report LA-13976-MS, Los Alamos National Laboratory, Los Alamos, NM (2003).

[9] K. Worden, C. Farrar, G. Manson, G. Park, The fundamental axioms of structural health monitoring, Proceedings of the Royal Society A: Mathematical, Physical and Engineering Science 463 (2082) (2007) 1639-1664.

[10] S. Doebling, C. Farrar, M. Prime, D. Shevitz, Damage identification and health monitoring of structural and mechanical systems from changes in their vibration characteristics: a literature review, Report LA-13070-MS, Los Alamos National Laboratory, Los Alamos, NM (1996).

[11] S. W. Doebling, C. R. Farrar, M. B. Prime, A summary review of vibration-based damage identification methods, The Shock and Vibration Digest 30 (2) (1998) 91-105.

[12] E. Carden, P. Fanning, Vibration based condition monitoring: A review, Structural Health Monitoring 3 (4) (2004) $355-377$.

[13] J. Brownjohn, A. De Stefano, Y.-L. Xu, H. Wenzel, A. Aktan, Vibration-based monitoring of civil infrastructure: challenges and successes, Journal of Civil Structural Health Monitoring 1 (3-4) (2011) 79-95.

[14] A. Deraemaeker, K. Worden (Eds.), New trends in vibration based structural health monitoring, Springer, Vienna, Austria, 2010.

[15] A. Rytter, Vibration based inspection of civil engineering structures, Ph.D. thesis, Aalborg University (1993).

[16] Y. Ben-Haim, S. Cogan, L. Sanseigne, Usability of mathematical models in mechanical decision processes, Mechanical Systems and Signal Processing 12 (1) (1998) 121-134.

[17] A. Deraemaeker, P. Ladevèze, P. Leconte, Reduced bases for model updating in structural dynamics based on constitutive relation error, Computer Methods in Applied Mechanics and Engineering 191 (2002) 2427-2444.

[18] A. Deraemaeker, P. Ladevèze, T. Romeuf, Model validation in the presence of uncertain experimental data, Engineering Computations 21 (8) (2004) 808-833.

[19] P. Ladevèze, G. Puel, A. Deraemaeker, T. Romeuf, Validation of structural dynamics models containing uncertainties, Mechanical Systems and Signal Processing 195 (2006) 373-393.

[20] P.-E. Charbonnel, P. Ladevèze, F. Louf, C. Le Noac'h, A robust CRE-based approach for model updating using in situ measurements, Computers and Structures 129 (2013) 63-73.

[21] J. Maeck, Damage assessment of civil engineering structures by vibration monitoring, Ph.D. thesis, Department of Civil Engineering, KU Leuven (2003).

[22] E. Reynders, System identification methods for (operational) modal analysis: review and comparison, Archives of Computational Methods in Engineering 19 (1) (2012) 51-124. URL http://dx.doi.org/10.1007/s11831-012-9069-x

[23] T. Toksoy, A. Aktan, Bridge-condition assessment by modal flexibility, Experimental Mechanics 34 (3) (1994) $271-278$.

[24] A. Pandey, M. Biswas, Damage detection in structures using changes in flexibility, Journal of Sound and Vibration 169 (1) (1994) 3-17. 
[25] F. Catbas, M. Gul, J. Burkett, Damage assessment using flexibility and flexibility-based curvature for structural health monitoring, Smart Materials and Structures 17 (1) (2008) 15-24.

[26] B. Jaishi, W.-X. Ren, Damage detection by finite element model updating using modal flexibility residual, Journal of Sound and Vibration 290 (1-2) (2006) 369-387.

[27] A. Pandey, M. Biswas, M. Samman, Damage detection from changes in curvature mode shapes, Journal of Sound and Vibration 145 (2) (1991) 321-332.

[28] M. Abdel Wahab, G. De Roeck, Damage detection in bridges using modal curvatures: application to a real damage scenario, Journal of Sound and Vibration 226 (2) (1999) 217-235. URL http://dx.doi.org/10.1006/jsvi.1999.2295

[29] Z. Y. Shi, S. S. Law, L. M. Zhang, Structural damage detection from modal strain energy change, Journal of Engineering Mechanics 126 (12) (2000) 1216-1223.

[30] B. Jaishi, W.-X. Ren, Finite element model updating based on eigenvalue and strain energy residuals using multiobjective optimisation technique, Mechanical Systems and Signal Processing 21 (5) (2007) 2295-2317.

[31] M. Imregun, W. Visser, D. Ewins, Finite-element model updating using frequency-response function data - 1. theory and initial investigation, Mechanical Systems and Signal Processing 9 (2) (1995) 187-202.

[32] S. Lammens, Frequency response based validation of dynamic structural finite element models, Phd thesis, KU Leuven, Belgium (1995).

[33] A. Esfandiari, F. Bakhtiari-Nejad, A. Rahai, M. Sanayei, Structural model updating using frequency response function and quasi-linear sensitivity equation, Journal of Sound and Vibration 326 (3-5) (2009) 557-573.

[34] Y. Haralampidis, C. Papadimitriou, M. Pavlidou, Multi-objective framework for structural model identification, Earthquake Engineering and Structural Dynamics 34 (2005) 665-685.

[35] K. Christodoulou, C. Papadimitriou, Structural identification based on optimally weighted modal residuals, Mechanical Systems and Signal Processing 21 (2007) 4-23.

[36] H. Schlune, M. Plos, K. Gylltoft, Improved bridge evaluation through finite element model updating using static and dynamic measurements, Engineering Structures 31 (7) (2009) 1477-1485.

[37] A. Björk, Numerical methods for least squares problems, SIAM Society for Industrial and Applied Mathematics, Philadelphia, USA, 1996.

[38] P. E. Gill, W. Murray, Algorithms for the solution of the nonlinear least squares problem, SIAM Journal on Numerical Analysis 15 (1978) 977-992.

[39] M. I. Friswell, J. E. Mottershead, Physical understanding of structures by model updating, in: Proceedings of COST F3 International Conference on Structural System Identification, Kassel, Germany, 2001, pp. 81-96.

[40] A. Teughels, J. Maeck, G. De Roeck, Damage assessment by FE model updating using damage functions, Computers and Structures 80 (25) (2002) 1869-1879. URL http://dx.doi.org/10.1016/S0045-7949(02)00217-1

[41] P. Hansen, Rank-deficient and discrete ill-posed problems, SIAM, 1998.

[42] A. Neumaier, Solving ill-conditioned and singular linear systems: A tutorial on regularization, SIAM Review 40 (3) (1998) 636-666.

[43] B. Titurus, M. Friswell, Regularization in model updating, International Journal for Numerical Methods in Engineering 75 (2008) 440-478.

[44] E. Reynders, G. De Roeck, Reference-based combined deterministic-stochastic subspace identification for experimental and operational modal analysis, Mechanical Systems and Signal Processing 22 (3) (2008) 617-637. URL http://dx.doi.org/10.1016/j.ymssp.2007.09.004

[45] The MathWorks, MATLAB Optimization Toolbox User's Guide (2011).

[46] D. Ghosh, R. Ghanem, J. Red-Horse, Analysis of eigenvalues and modal interaction of stochastic systems, AIAA Journal 43 (10) (2005) 2196-2201.

[47] R. Fox, M. Kapoor, Rate of change of eigenvalues and eigenvectors, AIAA Journal 6 (12) (1968) $2426-2429$.

[48] R. Nelson, Simplified calculation of eigenvector derivatives, AIAA Journal 14 (9) (1976) 1201-1205.

[49] C. Papadimitriou, E. Ntotsios, Structural model updating using vibration measurements, in: M. Papadrakakis, N. Lagaros, M. Fragiadakis (Eds.), Proceedings of the ECCOMAS Thematic Conference on Computational Methods in Structural Dynamics and Earthquake Engineering COMPDYN2009, Rhodes, Greece, 2009.

[50] W. Walker, P. Harremoes, J. Rotmans, J. van der Sluijs, M. van Asselt, P. Janssen, M. K. von Krauss, Defining uncertainty: A conceptual basis for uncertainty management in model-based decision support, Integrated Assessment 4 (1) (2003) 5-17.

[51] A. Der Kiureghian, O. Ditlevsen, Aleatory or epistemic? Does it matter?, Structural Safety 31 (2) (2009) 105-112.

[52] C. Soize, Generalized probabilistic approach of uncertainties in computational dynamics using random matrices and polynomial chaos decompositions, International Journal for Numerical Methods in Engineering 81 (8) (2010) 939-970.

[53] C. Soize, Stochastic models of uncertainties in computational structural dynamics and structural acoustics, Part of the CISM course on Nondeterministic Mechanics, Udine, Italy (May 2011).

[54] M. C. Kennedy, A. O. O'Hagan, Bayesian calibration of computer models, Journal of the Royal Statistical Society. Series B (Methodological) 63 (3) (2001) 425-464.

[55] J. Legault, R. Langley, J. Woodhouse, Physical consequences of a nonparametric uncertainty model in structural dynamics, Journal of Sound and Vibration 331 (25) (2012) 5469-5487.

[56] A. O'Hagan, J. Oakley, Probability is perfect, but we can't elicit it perfectly, Reliability Engineering and System Safety 85 (2004) 239-248.

[57] E. Reynders, R. Pintelon, G. De Roeck, Uncertainty bounds on modal parameters obtained from Stochastic Subspace 
Identification, Mechanical Systems and Signal Processing 22 (4) (2008) 948-969.

URL http://dx.doi.org/10.1016/j.ymssp.2007.10.009

[58] G. Schuëller, Computational stochastic mechanics - recent advances, Computers and Structures 79 (2001) $2225-2234$.

[59] G. Schuëller, Developments in stochastic structural mechanics, Archive of Applied Mechanics 75 (10-12) (2006) 755-773.

[60] G. Schuëller, On the treatment of uncertainties in structural mechanics and analysis, Computers and Structures 85 (5-6) (2007) 235-243.

[61] G. Schuëller, H. Pradlwarter, Uncertain linear systems in dynamics: Retrospective and recent developments by stochastic approaches, Engineering Structures 31 (11) (2009) 2507-2517.

[62] G. Schuëller, Special issue: Computational methods in stochastic mechanics and reliability analysis - Preface, Computer Methods in Applied Mechanics and Engineering 194 (12-16) (2005) iii-vii.

[63] G. Schuëller, Uncertainties in structural mechanics and analysis - computational methods: Preface, Computers and Structures 83 (14) (2005) 1031-1032.

[64] G. Deodatis, P. Spanos, Special issue - 5th International Conference on Computational Stochastic Mechanics - Preface, Probabilistic Engineering Mechanics 23 (2-3) (2008) 103.

[65] R. Ghanem, P. Spanos, Stochastic finite elements: a spectral approach, Springer-Verlag, New York, 1991.

[66] G. Stefanou, The stochastic finite element method: Past, present and future, Computer Methods in Applied Mechanics and Engineering 198 (9-12) (2009) 1031-1051.

[67] C. Soize, A nonparametric model of random uncertainties for reduced matrix models in structural dynamics, Probabilistic Engineering Mechanics 15 (2000) 277-294.

[68] C. Soize, A comprehensive overview of a non-parametric probabilistic approach of model uncertainties for predictive models in structural dynamics, Journal of Sound and Vibration 288 (2005) 623-652.

[69] M. Mehta, Random Matrices, 3rd Edition, Elsevier, San Diego, CA, 2004.

[70] C. Soize, Stochastic modeling of uncertainties in computational structural dynamics - recent theoretical advances, Journal of Sound and VibrationDoi:10.1016/j.jsv.2011.10.010.

[71] G. Casella, R. Berger, Statistical Inference, 2nd Edition, Duxbury Press, 2001.

[72] J. V. Beck, K. J. Arnold, Parameter Estimation in Engineering and Science, Wiley and Sons, New York, 1977.

[73] T. Saito, S. Mase, K. Morita, A probabilistic approach to structural damage estimation, Structural Control and Health Monitoring 12 (3-4) (2005) 283-299, International Workshop on Advanced Sensors, Structural Health Monitoring, and Smart Structures, Keio Univ, Yokohama, JAPAN, NOV 10-11, 2003.

[74] N. Hernández, R. Biscay, I. Talavera, A non-Bayesian predictive approach for statistical calibration, Journal of Statistical Computation and Simulation 82 (4) (2012) 529-545.

[75] J. Xia, H. Hao, Statistical damage identification of structures with frequency changes, Journal of Sound and Vibration 263 (4) (2003) 853-870.

[76] H. Khodaparast, J. Mottershead, M. Friswell, Perturbation methods for the estimation of parameter variability in stochastic model updating, Mechanical Systems and Signal Processing 22 (8) (2008) 1751-1773.

[77] C. Mares, J. Mottershead, M. Friswell, Stochastic model updating: Part 1 - theory and simulated example, Mechanical Systems and Signal Processing 20 (2006) 1674-1695.

[78] J. Fonseca, M. Friswell, J. Mottershead, A. Lees, Uncertainty identification by the maximum likelihood method, Journal of Sound and Vibration 288 (2005) 587-599.

[79] Y. Govers, M. Link, Stochastic model updating - covariance matrix adjustments from uncertain experimental modal data, Mechanical Systems and Signal Processing 24 (3) (2010) 696-706.

[80] E. Jacquelin, S. Adhikari, M. Friswell, A second-moment approach for direct probabilistic model updating in structural dynamics, Mechanical Systems and Signal Processing 29 (SI) (2012) 262-283.

[81] C. Desceliers, C. Soize, R. Ghanem, Identification of chaos representations of elastic properties of random media using experimental vibration tests, Computational Mechanics 39 (6) (2007) 831-838.

[82] R. Ghanem, A. Doostan, J. Red-Horse, A probabilistic construction of model validation, Computer Methods in Applied Mechanics and Engineering 197 (29-32) (2008) 2585-2595.

[83] L. Mehrez, A. Doostan, D. Moens, D. Vandepitte, Stochastic identification of composite material properties from limited experimental databases, Part II: Uncertainty modelling, Mechanical Systems and Signal Processing 27 (2012) $484-498$.

[84] B. Goller, H. Pradlwarter, G. Schuëller, Robust model updating with insufficient data, Computer Methods in Applied Mechanics and Engineering 198 (34-40) (2009) 3096-3104.

[85] J. Beck, L. Katafygiotis, Updating models and their uncertainties. I: Bayesian statistical framework, ASCE Journal of Engineering Mechanics 124 (4) (1998) 455-461.

[86] S. Rao, L. Berke, Analysis of uncertain structural systems using interval analysis, AIAA JOURNAL 35 (4) (1997) $727-735$.

[87] R. Muhanna, R. Mullen, Uncertainty in mechanics problems - Interval-based approach, Journal of Engineering Mechanics 127 (6) (2001) 557-566.

[88] D. Moens, D. Vandepitte, Interval sensitivity theory and its application to frequency response envelope analysis of uncertain structures, Computer Methods in Applied Mechanics and Engineering 196 (21-24) (2007) 2486-2496.

[89] S. Gabriele, C. Valente, An interval-based technique for FE model updating, International Journal of Reliability and Safety 3 (2009) 79-103.

[90] O. García, J. Vehí, J. Campos e Matos, A. Henriques, J. Casas, Structural assessment under uncertain parameters via interval analysis, Journal of Computational and Applied Mathematics 217 (1) (2008) 43-52.

[91] H. Khodaparast, J. Mottershead, K. Badcock, Interval model updating with irreducible uncertainty using the kriging predictor, Mechanical Systems and Signal Processing 25 (4) (2011) 1204-1226. 
[92] H. Khodaparast, J. Mottershead, Y. Govers, M. Link, Interval model updating of the AIRMOD structure, in: P. Sas, D. Moens, S. Jonckheere (Eds.), Proceedings of ISMA 2012 International Conference on Noise and Vibration Engineering, Leuven, Belgium, 2012, pp. 4717-4732, cD-ROM.

[93] Y. Ben-Haim, I. Elishakoff, Convex models of uncertainty in applied mechanics, Elsevier Science, Amsterdam, 1990.

[94] J. Hu, Z. Qiu, Non-probabilistic convex models and interval analysis method for dynamic response of a beam with bounded uncertainty, Applied Mathematical Modelling 34 (2010) 725-734.

[95] M. Lombardi, R. T. Haftka, Anti-optimization technique for structural design under load uncertainties, Computer Methods in Applied Mechanics and Engineering 157 (1-2) (1998) 19-31.

[96] S. Ferson, W. Tucker, Sensitivity analysis using probability bounding, Reliability Engineering and System Safety 91 (1011) (2006) 1435-1442.

[97] L. V. Utkin, An uncertainty model of structural reliability with imprecise parameters of probability distributions, Zeitschrift für Angewandte Mathematik und Mechanik 84 (10-11) (2004) 688-699.

[98] K. Weichselberger, The theory of interval-probability as a unifying concept for uncertainty, International Journal of Approximate Reasoning 24 (2-3) (2000) 149 - 170.

[99] A. P. Dempster, Upper and lower probabilities induced by a multivalued mapping, The Annals of Mathematical Statistics 38 (2) (1967) 325-339.

[100] G. Shafer, A mathematical theory of evidence, Princeton University Press, New Jersey, 1976.

[101] H. Kwakernaak, Fuzzy random variables - I. Definitions and theorems, Information Sciences 15 (1) (1978) 1-29.

[102] B. Möller, Fuzzy randomness - a contribution to imprecise probabilities, Zeitschrift für Angewandte Mathematik und Mechanik 84 (10-11) (2004) 754-764.

[103] B. Möller, M. Beer, Fuzzy Randomness - Uncertainty in Civil Engineering and Computational Mechanics, Springer, Berlin, 2004.

[104] R. Viertl, Statistical methods for fuzzy data, John Wiley \& Sons, Chichester, U.K., 2011.

[105] T. Bayes, An essay towards solving a problem in the doctrine of chances, Philosophical Transactions of the Royal Society 53 (1763) 370-418.

[106] P.-S. Laplace, Memoir on the probability of the causes of events, Statistical Science 1 (3) (1774/1986) 364-378.

[107] R. Cox, Probability, frequency and reasonable expectation, American Journal of Physics 14 (1) (1946) 1-13.

[108] E. Jaynes, Probability Theory. The Logic of Science, Cambridge University Press, Cambridge, UK, 2003.

[109] L. Katafygiotis, J. Beck, Updating models and their uncertainties. II: Model identifiability, ASCE Journal of Engineering Mechanics 124 (4) (1998) 463-467.

[110] L. Katafygiotis, P. C., H. Lam, A probabilistic approach to structural model updating, Soil Dynamics and Earthquake Engineering 17 (1998) 495-507.

[111] J. L. Beck, Bayesian system identification based on probability logic, Structural Control and Health Monitoring 17 (7) (2010) 825-847.

[112] K. Mosegaard, A. Tarantola, Probabilistic approach to inverse problems, in: International Handbook of Earthquake \& Engineering Seismology, Part A, Academic Press, 2002, pp. 237-265.

[113] A. Tarantola, Inverse problem theory and methods for model parameter estimation, SIAM, Philadelphia, USA, 2005.

[114] G. Box, G. Tiao, Bayesian inference in statistical analysis, Addison-Wesley, 1973.

[115] V. Peterka, Bayesian system identification, Automatica 17 (1) (1981) 41-53.

[116] M. Vanik, J. Beck, S. Au, Bayesian probabilistic approach to structural health monitoring, ASCE Journal of Engineering Mechanics 126 (7) (2000) 738-745.

[117] J. Beck, S.-K. Au, W. Vanik, Monitoring structural health using a probabilistic measure, Computer-Aided Civil and Infrastructure Engineering 16 (2001) 1-11.

[118] K.-V. Yuen, Bayesian methods for structural dynamics and civil engineering, 1st Edition, John Wiley \& Sons, Singapore, 2010.

[119] K.-V. Yuen, J. Beck, L. Katafygiotis, Efficient model updating and health monitoring methodology using incomplete modal data without mode matching, Structural Control and Health Monitoring 13 (2006) 91-107.

[120] K.-V. Yuen, J. Beck, L. Katafygiotis, Unified probabilistic approach for model updating and damage detection, Journal of applied mechanics 73 (4) (2006) 555-564

[121] J. Ching, J. L. Beck, New Bayesian model updating algorithm applied to a structural health monitoring benchmark, Structural Health Monitoring 4 (3) (2004) 313-332.

[122] J. Ching, M. Muto, J. Beck, Structural model updating and health monitoring with incomplete modal data using Gibbs sampler, Computer-Aided Civil and Infrastructure Engineering 21 (2006) 242-257.

[123] J. Ching, M. Muto, J. L. Beck, Bayesian linear structural model updating using Gibbs sampler with modal data, in: G. Augusti, G. Schuëller, M. Ciampoli (Eds.), Proceedings of the 9th International Conference On Structural Safety And Reliability: ICOSSAR 2005, Rome, Italy, 2005.

[124] S. Cheung, J. Beck, Bayesian model updating using hybrid monte carlo simulation with application to structural dynamic models with many uncertain parameters, ASCE Journal of Engineering Mechanics 134 (4) (2006) $243-255$.

[125] C. Papadimitriou, J. Beck, L. Katafygiotis, Updating robust reliability using structural test data, Probabilistic Engineering Mechanics 16 (2) (2001) 103-113.

[126] J. Choi, D. An, J. Won, Bayesian approach for structural reliability analysis and optimization using the Kriging dimension reduction method, Journal of Mechanical Design 132 (5) (2010) 1-11.

[127] H. Sohn, K. Law, A Bayesian probabilistic approach for structure damage detection, Earthquake Engineering and Structural Dynamics 26 (12) (1997) 1259-1281.

[128] H. Sohn, K. Law, Bayesian probabilistic damage detection of a reinforced-concrete bridge column, Earthquake Engineering 
and Structural Dynamics 29 (8) (2000) 1131-1152.

[129] J. Beck, Robust stochastic predictions of dynamic response during and monitoring of structures, Procedia Engineering 14 (2011) 32-40, the Proceedings of the Twelfth East Asia-Pacific Conference on Structural Engineering and Construction.

[130] J. Beck, K.-V. Yuen, Model selection using response measurements: Bayesian probabilistic approach, ASCE Journal of Engineering Mechanics 130 (2) (2004) 192-203.

[131] K.-V. Yuen, Recent developments of Bayesian model class selection and applications in civil engineering, Structural Safety 32 (5) (2010) 338-346.

[132] E. Jaynes, Prior probabilities, IEEE Transactions on Systems Science and Cybernetics sec-4 (3) (1968) $227-241$.

[133] J. M. Bernardo, Reference posterior distributions for Bayesian inference, Journal of the Royal Statistical Society. Series B (Methodological) 41 (2) (1979) 113-147.

134] J. O. Berger, J. M. Bernardo, D. Sun, The formal definition of reference priors, The Annals of Statistics 37 (2) (2009) 905-938.

[135] P. Diaconis, D. Ylvisaker, Conjugate priors for exponential families, The Annals of Statistics 7 (2) (1979) 269-281.

[136] H. Jeffreys, An invariant form for the prior probability in estimation problems, Proceedings of the Royal Society A: Mathematical, Physical and Engineering Sciences 186 (1007) (1946) 453-461.

[137] E. Jaynes, Information theory and statistical mechanics, The Physical Review 106 (4) (1957) 620-630.

[138] E. Jaynes, Information theory and statistical mechanics II, The Physical Review 108 (2) (1957) 171-190.

[139] S. Gull, Bayesian inductive inference and maximum entropy, Maximum-Entropy and Bayesian methods in Science and Engineering 1 (1988) 53-74.

[140] C. Soize, Maximum entropy approach for modeling random uncertainties in transient elastodynamics, Journal of the Acoustical Society of America 109 (5) (2001) 1979-1996.

[141] C. Soize, Construction of probability distributions in high dimensions using the maximum entropy principle: applications to stochastic processes, random fields and random matrices, International Journal for Numerical Methods in Engineering 75 (2008) 1583-1611.

[142] C. Soize, Probabilités et modélisation des incertitudes: éléments de base et concepts fondamentaux, Handed out at the séminaire de formation de l'école doctorale MODES, Paris (May 2003).

[143] K. Christodoulou, Methodology for structural identification and damage detection, Ph.D. thesis, University of Thessaly, Greece (2006)

[144] C. Papadimitriou, Bayesian updating of weight values uncertainties in weighted residuals methods for model updating and response predictions, in: Proceedings of the 24th International Conference On Sound and Vibration in Engineering and 3rd International conference on Uncertainty in Structural Dynamics: ISMA2010 including USD2010, Leuven, Belgium, 2010, pp. 5207-5222.

[145] E. L. Zhang, P. Feissel, J. Antoni, A comprehensive Bayesian approach for model updating and quantification of modeling errors, Probabilistic Engineering Mechanics 26 (2011) 550-560.

[146] E. Simoen, C. Papadimitriou, G. Lombaert, On prediction error correlation in Bayesian model updating, Journal of Sound and Vibration 332 (18) (2013) 4136-4152.

[147] P. Gardoni, K. Mosalam, A. der Kiureghian, Probabilistic seismic demand models and fragility estimates for RC bridges, Journal of Earthquake Engineering 7 (1) (2003) 79-106.

[148] M. Muto, J. Beck, Bayesian updating and model class selection for hysteretic structural models using stochastic simulation, Journal of Vibration and Control 14 (1-2) (2008) 7-34.

[149] E. Ntotsios, C. Papadimitriou, P. Panetsos, G. Karaiskos, K. Perros, P. Pardikaris, Bridge health monitoring system based on vibration measurements, Bulletin of Earthquake Engineering 7 (2) (2009) 469-483.

[150] L. Mthembu, T. Marwala, M. I. Friswell, S. Adhikari, Model selection in finite element model updating using the Bayesian evidence statistic, Mechanical Systems and Signal Processing 25 (2011) 2399-2412.

[151] E. Simoen, Uncertainty quantification in finite element model updating, Ph.D. thesis, Department of Civil Engineering, KU Leuven (2013).

[152] E. Simoen, B. Moaveni, J. Conte, G. Lombaert, Uncertainty quantification in the assessment of progressive damage in a seven-story full-scale building slice, ASCE Journal of Engineering Mechanics 139 (12) (2013) 1818-1830.

[153] R. Kalman, A new approach to linear filtering and prediction problems, Journal of Basic Engineering, Transactions of the ASME 82D (1960) 35-45.

[154] A. Jazwinski, Stochastic processes and filtering theory, Academic Press New York, 1970.

[155] C. Papadimitriou, J. Beck, L. Katafygiotis, Asymptotic expansions for reliability and moments of uncertain systems, ASCE Journal of Engineering Mechanics 123 (12) (1997) 1219-1229.

[156] D. Gamerman, Markov Chain Monte Carlo: stochastic simulation for Bayesian inference, Chapman \& Hall, London, 1997.

[157] K. Mosegaard, A. Tarantola, Monte Carlo sampling of solutions to inverse problems, Journal of Geophysical Research 100 (1995) 12431-12447.

[158] L. Tierney, Markov chains for exploring posterior distributions, The Annals of Statistics 22 (4) (1994) $1701-1728$.

[159] N. Metropolis, A. Rosenbluth, M. Rosenbluth, A. Teller, Equation of state calculations by fast computing machines, Journal of Chemical Physics 21 (6) (1953) 1087-1092.

[160] H. Haario, E. Saksman, J. Tamminen, An adaptive Metropolis algorithm, Bernouilli 7 (2) (2001) $223-242$.

[161] L. Tierney, A. Mira, Some adaptive Monte Carlo methods for Bayesian inference, Statistics in Medicine 18 (1999) 25072515.

[162] P. Green, A. Mira, Delayed rejection in reversible jump Metropolis-Hastings, Biometrika 88 (4) (2001) $1035-1053$.

[163] A. Mira, Ordering and Improving the Performance of Monte Carlo Markov Chains, Statistical Science 16 (4) (2001) 
340-350.

[164] H. Haario, M. Laine, A. Mira, E. Saksman, DRAM: Efficient adaptive MCMC, Statistics and Computing 16 (4) (2006) 339-354.

[165] S. Au, C. Papadimitriou, J. Beck, Reliability of uncertain dynamical systems with multiple design points, Structural Safety 21 (1999) 113-133.

[166] J. Beck, S.-K. Au, Bayesian updating of structural models and reliability using Markov Chain Monte Carlo simulation, ASCE Journal of Engineering Mechanics 128 (4) (2002) 380-391.

[167] J. Ching, Y.-C. Chen, Transitional Markov Chain Monte Carlo method for Bayesian model updating, model class selection, and model averaging, ASCE Journal of Engineering Mechanics 133 (7) (2007) 816-832.

[168] P. Angelikopoulos, C. Papadimitriou, P. Koumoutsakos, Bayesian uncertainty quantification and propagation in molecular dynamics simulations: A high performance computing framework, Journal of Chemical Physics 137 (4).

[169] C. J. F. Ter Braak, A Markov Chain Monte Carlo version of the genetic algorithm differential evolution: easy Bayesian computing for real parameter spaces, Statistics and Computing 16 (3) (2006) 239-249.

[170] J. Vrugt, C. J. F. ter Braak, C. G. H. Diks, B. A. Robinson, J. M. Hyman, D. Higdon, Accelerating Markov Chain Monte Carlo Simulation by Differential Evolution with Self-Adaptive Randomized Subspace Sampling, International Journal of Nonlinear Sciences and Numerical Simulation 10 (3) (2009) 273-290.

[171] B. Möller, M. Beer, Engineering computation under uncertainty - capabilities of non-traditional models, Computers and Structures 86 (2008) 1024-1041.

[172] W. Oberkampf, S. DeLand, B. Rutherford, K. Diegert, K. Alvin, Error and uncertainty in modeling and simulation, Reliability Engineering and System Safety 75 (2002) 333-357.

[173] B. Efron, 1977 Rietz Lecture - Bootstrap methods - Another look at the Jackknife, Annals of Statistics 7 (1) (1979) $1-26$.

[174] J. Helton, J. Johnson, W. Oberkampf, An exploration of alternative approaches to the representation of uncertainty in model predictions, Reliability Engineering and System Safety 85 (2004) 39-71.

[175] M. Oberguggenberger, Introductory remarks: mathematical models of uncertainty, Zeitschrift für Angewandte Mathematik und Mechanik 84 (10-11) (2004) 661-665.

[176] T. J. Ross, M. J. Booker, W. J. Parkinson, Fuzzy logic and probability applications - Bridging the gap, ASA-SIAM, Philadelphia, 2002.

[177] A. Bernardini, F. Tonon, Bounding uncertainty in civil engineering - theoretical background, Springer, 2010.

[178] H.-R. Bae, R. V. Grandhi, R. A. Canfield, Epistemic uncertainty quantification techniques including evidence theory for large-scale structures, Computers and Structures 82 (13-14) (2004) 1101-1112.

[179] S. Ferson, C. Joslyn, J. Helton, W. Oberkampf, K. Sentz, Summary from the epistemic uncertainty workshop: consensus amid diversity, Reliability Engineering and System Safety 85 (2004) 355-369.

[180] W. L. Oberkampf, J. C. Helton, C. A. Joslyn, S. F. Wojtkiewicz, S. Ferson, Challenge problems: uncertainty in system response given uncertain parameters, Reliability Engineering and System Safety 85 (2004) 11-19.

[181] I. Elishakoff, Essay on uncertainties in elastic and viscoelastic structures: From A. M. Freudenthal's criticisms to modern convex modeling, Computers and Structures 56 (6) (1995) 871-895.

[182] I. Elishakoff, Safety factors and reliability: friends or foes?, Kluwer Academic Publishers, Dordrecht, 2004.

[183] I. Elishakoff, M. Ohsaki, Optimization and anti-optimization of structures under uncertainty, Imperial College Press, 2010 .

[184] R. S. Langley, Unified approach to probabilistic and possibilistic analysis of uncertain systems, Journal of Engineering Mechanics 126 (11) (2000) 1163-1172.

[185] M. Oberguggenberger, G. Schueller, K. Marti, Special edition: Fuzzy sets, imprecise probability, and stochastics in engineering, Zeitschrift für Angewandte Mathematik und Mechanik 84 (10-11) (2004) 659-660.

[186] L. A. Zadeh, Fuzzy sets, Information and Control 8 (3) (1965) 338-353.

[187] D. Dubois, H. Prade, The three semantics of fuzzy sets, Fuzzy Sets and Systems 90 (2) (1997) 141-150.

[188] D. Degrauwe, Uncertainty propagation in structural analysis by fuzzy numbers, Ph.D. thesis, Department of Civil Engineering, KU Leuven (2007).

[189] M. Oberguggenberger, Combined methods, Part of the CISM course on Nondeterministic Mechanics, Udine, Italy (May 2011).

[190] A. Kaufmann, Introduction to the theory of fuzzy subsets, Vol. 1, Academic Press, New York, 1975.

[191] L. A. Zadeh, Fuzzy sets as a basis for a theory of possibility, Fuzzy Sets and Systems 1 (1) (1978) 3-28.

[192] H. J. Zimmermann, Fuzzy set theory and its applications, 2nd Edition, Kluwer Academic Publishers, Boston, 1991.

[193] M. Beer, Uncertain structural design based on nonlinear structural analysis, Zeitschrift für Angewandte Mathematik und Mechanik 84 (10-11) (2004) 740-753.

[194] M. Beer, M. Liebsher, Designing robust structures - A nonlinear simulation based approach, Computers and Structures 86 (2008) 1102-1122.

[195] Y. Ben-Haim, Info-gap value of information in model updating, Mechanical Systems and Signal Processing 15 (3) (2001) 457-474.

[196] B. Möller, W. Graf, M. Beer, Fuzzy structural analysis using $\alpha$-level optimization, Computational Mechanics 26 (2000) $547-565$.

[197] R. Muhanna, R. Mullen, Formulation of fuzzy finite-element methods for solid mechanics problems, Computer-Aided Civil and Infrastructure Engineering 14 (2) (1999) 107-117.

[198] M. V. Rama Rao, R. Ramesh Reddy, Fuzzy finite element analysis of structures with uncertainty in load and material properties, ASCE Journal of Structural Engineering 33 (2) (2006) 129-137. 
[199] K. K. Choi, L. Du, B. D. Youn, A new fuzzy analysis method for possibility-based design optimization, in: Proceedings of the 10th AIAA/ISSMO Conference, 2004.

[200] H. A. Jensen, Structural optimal design of systems with imprecise properties: a possibilistic approach, Advances in Engineering Software 32 (12) (2001) 937-948.

[201] F. Massa, T. Tison, B. Lallemand, A fuzzy procedure for the static design of imprecise structures, Computer Methods in Applied Mechanics and Engineering 195 (9-12) (2006) 925-941.

[202] D. Moens, D. Vandepitte, A fuzzy finite element procedure for the calculation of uncertain frequency-response functions of damped structures: Part 1 procedure, Journal of Sound and Vibration 288 (3) (2005) 431-462.

[203] D. Degrauwe, G. De Roeck, G. Lombaert, Fuzzy frequency response function of a composite floor subject to uncertainty by application of the G $\alpha \mathrm{D}$ algorithm, in: C. Mota Soares (Ed.), Proceedings of the 3rd European Conference on Computational Mechanics, Lisbon, Portugal, 2006, cD-ROM.

[204] S. Adhikari, R. Chowdhury, M. I. Friswell, High dimensional model representation method for fuzzy structural dynamics, Journal of Sound and Vibration 330 (7) (2011) 1516-1529.

[205] R. Chowdhury, S. Adhikari, Fuzzy parametric uncertainty analysis of linear dynamical systems: A surrogate modeling approach, Mechanical Systems and Signal Processing 32 (2012) 5-17.

[206] H. Khodaparast, J. Mottershead, K. Badcock, Propagation of structural uncertainty to linear aeroelastic stability, Computers and Structures 88 (3-4) (2010) 223-236.

[207] L. V. Utkin, S. V. Gurov, A general formal approach for fuzzy reliability analysis in the possibility context, Fuzzy Sets and Systems 83 (2) (1996) 203-213.

[208] M. B. Anoop, K. B. Rao, T. V. S. R. A. Rao, Application of fuzzy sets for estimating service life of reinforced concrete structural members in corrosive environments, Engineering Structures 24 (9) (2002) 1229-1242.

[209] F. Biondini, F. Bontempi, P. G. Malerba, Fuzzy reliability analysis of concrete structures, Computers and Structures $82(13-14)(2004) 1033-1052$.

[210] C. I. Giasi, P. Masi, C. Cherubini, Probabilistic and fuzzy reliability analysis of a sample slope near Aliano, Engineering Geology 67 (3-4) (2003) 391-402.

[211] M. Savoia, Structural reliability analysis through fuzzy number approach, with application to stability, Computers and Structures 80 (12) (2002) 1087-1102.

[212] T. Haag, S. González, M. Hanss, Model validation and selection based on inverse fuzzy arithmetic, Mechanical Systems and Signal Processing 32 (2012) 116-134.

[213] T. Haag, J. Herrmann, M. Hanss, Identification procedure for epistemic uncertainties using inverse fuzzy arithmetic, Mechanical Systems and Signal Processing 24 (7) (2010) 2021-2034.

[214] M. Hanss, An approach to inverse fuzzy arithmetic, in: Fuzzy Information Processing Society, 2003. NAFIPS 2003. 22nd International Conference of the North American Fuzzy Information Processing Society - NAFIPS 2003, Chicago, IL, USA, 2003, pp. 474-479.

[215] D. Degrauwe, G. Lombaert, G. De Roeck, Improving interval analysis in finite element calculations by means of affine arithmetic, Computers and Structures 88 (3-4) (2010) 247-254. URL http://dx.doi.org/10.1016/j.compstruc.2009.11.003

[216] J. Nocedal, S. Wright, Numerical Optimization, Springer, New York, USA, 1999.

[217] P. E. Gill, W. Murray, M. H. Wright, Practical Optimization, $11^{\text {th }}$ Edition, Academic Press Limited, San Diego, 1997.

[218] W. Dong, H. C. Shah, Vertex method for computing functions of fuzzy variables, Fuzzy Sets and Systems 24 (1) (1987) $65-78$.

[219] M. Hanss, The transformation method for the simulation and analysis of systems with uncertain parameters, Fuzzy Sets and Systems 130 (3) (2002) 277-289.

[220] M. Hanss, A. Klimke, On the reliability of the influence measure in the transformation method of fuzzy arithmetic, Fuzzy Sets and Systems 143 (3) (2004) 371-390.

[221] S. Donders, D. Vandepitte, J. V. d. Peer, W. Desmet, Assessment of uncertainty on structural dynamic responses with the short transformation method, Journal of Sound and Vibration 288 (3) (2005) 523-549.

[222] E. Untiedt, W. Lodwick, Using gradual numbers to analyze non-monotonic functions of fuzzy intervals, in: Fuzzy Information Processing Society, 2008. NAFIPS 2008. Annual Meeting of the North American, IEEE, 2008, pp. 1-6.

[223] D. Degrauwe, E. O. Arman, E. Reynders, G. De Roeck, G. Lombaert, An efficient fuzzy calculation algorithm with application to finite element model updating, in: P. Sas, M. De Munck (Eds.), Proceedings of ISMA2006 International Conference on Noise and Vibration Engineering, Leuven, Belgium, 2006, pp. 4105-4116.

[224] D. Jones, M. Schonlau, W. Welch, Efficient global optimization of expensive black-box functions, Journal of Global Optimization 13 (4) (1998) 455-492.

[225] M. Stein, Interpolation of spatial data: some theory for kriging, Springer, New York, 1999

[226] M. De Munck, D. Moens, W. Desmet, D. Vandepitte, A response surface based optimisation algorithm for the calculation of fuzzy envelope frfs of models with uncertain properties, Computers and Structures 86 (10) (2008) 1080?-1092.

[227] S. Corveleyn, S. Vandewalle, A polynomial response surface approach for the solution of fuzzy elliptical partial differential equations, in: G. De Roeck, G. Degrande, G. Lombaert, G. Müller (Eds.), Proceedings of the 8th International Conference on Structural Dynamics EURODYN 2011, Leuven, Belgium, 2011, pp. 3049-3055, cD-ROM.

[228] U. O. Akpan, T. S. Koko, I. R. Orisamolu, B. K. Gallant, Practical fuzzy finite element analysis of structures, Finite Elements in Analysis and Design 38 (2) (2001) 93-111.

[229] A. Klimke, B. Wohlmuth, Computing expensive multivariate functions of fuzzy numbers using sparse grids, Fuzzy Sets and Systems 154 (3) (2005) 432-453.

[230] G. Venter, R. Haftka, Using response surface approximations in fuzzy set based design optimization, Structural Opti- 
mization 18 (4) (1999) 218-227.

[231] J. E. Chen, K. N. Otto, Constructing membership functions using interpolation and measurement theory, Fuzzy Sets and Systems 73 (3) (1995) 313-327.

[232] A. L. Medaglia, S.-C. Fang, H. L. W. Nuttle, J. R. Wilson, An efficient and flexible mechanism for constructing membership functions, European Journal of Operational Research 139 (1) (2002) 84-95.

[233] R. Fullér, P. Majlender, On interactive fuzzy numbers, Fuzzy Sets and Systems 143 (3) (2004) 355-369.

[234] D. Degrauwe, G. Lombaert, G. De Roeck, Fuzzy frequency response function calculation with interactive fuzzy numbers, in: Proceedings of Leuven Symposium on Applied Mechanics in Engineering, Leuven, 2008, cD-ROM. 